\title{
A Fourier series solution for transient three-dimensional thermohaline convection in porous enclosures
}

\author{
Sara Tabrizi Nejad As ${ }^{1}$, Marwan Fahs s, ${ }^{1,}$, Behzad Ataie-Ashtiani ${ }^{2}$, Craig. T. Simmons ${ }^{2}$, di Chiara \\ Roupert Raphaël ${ }^{1}$, Anis Younes ${ }^{1}$ \\ ${ }^{1}$ Laboratoire d'Hydrologie et Geochemie de Strasbourg, University of Strasbourg/EOST/ENGEES, \\ CNRS, 1 Rue Blessig, 67084 Strasbourg, France \\ ${ }^{2}$ National Centre for Groundwater Research and Training \& College of Science and Engineering, \\ Flinders University, GPO Box 2100, Adelaide, SA 5001, Australia
}

Submitted to Water Resources Research

*Contact person: Marwan Fahs

E-mail: fahs@unistra.fr 


\begin{abstract}
Thermohaline convection (THC) in porous media is frequently investigated using the problem of porous enclosure. Most of the existing modeling-based studies are limited to 2D simulations, because $2 \mathrm{D}$ assumption is widely used to deal with computational requirement of $3 \mathrm{D}$ numerical solutions. Analytical solutions serve as an alternative to deal with computational requirement of numerical solutions. Existing analytical solutions of THC are mostly limited to 2D and also under steady-state regime. In this work, we develop a meshless 3D semi-analytical solution for the problem of THC in a porous box under crossed thermal and solute gradients, for both steadystate and transient regimes. The semi-analytical solution is developed using the Fourier series (FS) method applied to the vector potential form of the governing equations. The extension to transient solutions represents an important technical feature of this work, as the applications of the FS method to density-driven problems have been limited to steady-state conditions. The FS solution is validated against a finite element solution obtained using COMSOL Multiphysics. Numerical experiments show the worthiness of the developed FS solution as a benchmark because it clearly allows making distinction between different numerical techniques. The effects of governing parameters on three-dimensional THC have not been investigated previously. We perform a detailed parameter sensitivity analysis to address this gap. A vortex convective flow is observed and the orientation and intensity of the flow is sensitive to the gravity number. The increase in the temperature gradient reduces the salinity flux.
\end{abstract}

Keywords: Thermohaline convection; 3D semi-analytical solution; Crossed thermal and salinity gradients; Fourier series; benchmarking; COMSOL Multiphysics. 


\section{Introduction}

Thermal and solute variations through porous media are the main causes of density changes of fluid and may give rise to density-driven flow. This phenomenon is usually called thermohaline or thermosolutal convection (THC). When the flow is driven by both temperature and solute gradients the problem is also called double diffusive convection (DDC) (Zhao et al., 2008a). This phenomenon can be observed in several applications such as in geological carbon dioxide sequestration (Babaei and Islam, 2018; Islam et al., 2014), geothermal systems (Bao and Liu, 2019; Le Lous et al., 2015; van Lopik, et al., 2015), underground thermal energy storage (Cabeza et al., 2015), salt mining (Zechner et al., 2019), salt domes (Jamshidzadeh et al., 2015; Evans et al., 2015), groundwater management (love et al., 2007), soil contamination (Neild et al., 2008), and waste disposal and seawater intrusion (Langevin et al., 2010; Thorne et al., 2006).

THC studies in real systems at the field scale are increasingly reported in the literature. For instance, Yilmaz Turali and Simsek (2017) performed THC simulations of the Sorgun hydrothermal reservoir (Turkey). Jamshidzadeh et al. (2015) investigated THC near salt dome at Napoleonville Dome (USA). Sheldon et al. (2012) assessed the potential for thermal convection to occur in the Perth Basin in Australia. Schilling et al. (2013) used THC simulations to provide insight into the subsurface thermal regime of the same basin. Magri et al. (2012) investigated fault-induced seawater intrusion in a geothermal system. However, in most theoretical and academic research and in several engineering applications, THC is frequently investigated using the problem of a porous enclosure. This problem is widely used to understand the THC physical processes and as a common benchmark for numerical models and schemes. Unstable configurations of this problem, in which the fluid density decreases with depth (vertical thermal and solute gradients either opposing or cooperating each other), have been extensively studied 
for different purposes. For instance, Cooper et al. (2001) performed an experimental study to evaluate the effect of buoyancy ratio on the development of double-diffusive finger convection in a Hele-Shaw cell. Based on a rectangular porous enclosure, Islam et al. (2013) investigated double diffusive convection of CO2 in a brine saturated geothermal reservoir. Islam et al. (2014) extended their previous study to heterogeneous domains. Jamshidzadeh et al. (2013) used the modified thermohaline Elder problem to evaluate the effect of fluid dispersion on the THC. Fingering phenomena due to the DDC has been investigated in Hughes et al. (2005) and Musuuza et al. (2012). A square porous enclosure is considered in Babaei and Islam (2018) to study convective- reactive CO2 dissolution in aquifers and in Mansour et al. (2006) and Khadiri et al. (2010) to evaluate the Soret effect on the THC. Several works on unstable THC are concerned with the onset of convective flow, based on linear or nonlinear stability analysis (e.g. Jafari Raad et al., 2019; Nield and Kuznetsov, 2013; Javaheri et al., 2010). Stable configurations (horizontal thermal and solute gradients) of the problem of THC in a porous enclosure have also been widely investigated in the literature as they are important in many applications. In this context, several previous works performed parameter sensitivity analysis to understand the effect of governing parameters on the flow, heat, and mass processes and on the overall rate of heat and mass transfer (e.g. Trevisan and Bejan, 1986; Alavyoon 1993). Chamkha et al (2002) studied the THC in a rectangular porous enclosure with cooperating gradients and evaluate the effects of heat generation or absorption. Bennacer et al. (2001) investigated the effect of anisotropy on the average Nusselt and Sherwood numbers. The effect of anomalous fluid density was investigated in Sivasankaran et al. (2008). Mchirgui et al. (2012) studied entropy generation due to the THC. The influence of a local non-equilibrium state on the THC has been developed in Bera et al. (2014). Shao et al. (2016) developed a benchmark reference solution and investigated the effect 
of heterogeneity on THC. Most studies dealt with horizontal thermal and solute gradients either cooperating or opposing each other. Few studies investigated cases with crossed (verticalhorizontal) heat and mass gradients (Kalla et al., 2001; Mohamad and Bennacer, 2001, 2002; Mansour et al., 2006).

Despite the fact that THC processes are three-dimensional in nature, due to boundary conditions and/or domain heterogeneity, all of the works described above are limited by the assumption of two-dimensional flow. The $2 \mathrm{D}$ assumption is widely adopted to deal with computational complexity (computational cost, memory requirement, unphysical oscillations and convergence issues), as THC simulations require simultaneously solving the coupled nonlinear equations of flow, solute transport and heat transfer under variable fluid density. Regarding three-dimensional THC problems, the most studied configuration is the case of horizontal thermal and solute gradients. Based on numerical simulations and laboratory experiments, Sezai and Mohamad (1999) found that, for a certain range of parameters (Lewis and Rayleigh numbers), the convective flow is strictly three-dimensional. Stajnko et al. (2017) developed a 3D solution based on the boundary element method and investigated the effect of governing parameters on convective flow and rate of heat and mass transfer. Zhu et al. (2017) studied the influence of heterogeneity on entropy generation associated to the THC. Hadidi and Bennacer (2018) studied the THC in a bi-layered porous domain. Mohamad and Bennacer (2001) investigated the THC in a 3D porous enclosure subjected to vertical solute and horizontal heat gradients. They indicated that, even though the convective flow has a three-dimensional structure, the difference between three-and two-dimensional rate of mass and heat transfer is not significant. The cases considered in the literature dealt with specific boundary conditions for which the problem can be simplified to 2D. While most engineering applications and real-world problem of THC are unsteady or 
transient in nature, the existing works are limited to steady-state conditions. Although computational simulations have been conducted for 3D convection problems in the emerging computational geoscience field (Zhao et al., 2009, 2018), there is a need for developing new efficient and accurate methods to investigate 3D THD cases under transient conditions with higher Rayleigh numbers.

Analytical solutions can serve as an alternative to provide high accurate solutions and to avoid computational requirements of numerical solutions. They are helpful to provide insight on the physical processes as they are free of numerical errors. Analytical solutions are also important for benchmarking numerical codes and for the assessment of numerical schemes such as in the convergence analysis. However, analytical solutions are usually limited to specific boundary conditions and geometry and cannot be obtained without significant simplifications of the governing equations. Semi-analytical solutions combine the accuracy of analytical solutions with the flexibility of numerical solutions in solving the full mathematical models under complex and realistic boundary conditions. For the THC, Kalla et al. (2001) developed a 2D analytical solution based on the parallel flow approximation. Analytical solutions for 2D cases have been obtained in Trevisan and Bejan (1986) and Masuda et al. (2013) using the boundary layer approximation. Shao et al. (2015) developed a 2D semi-analytical solution for the full mathematical model (without any approximation), based on the Fourier series method (FS). Mostly, the existing analytical or semi-analytical solutions are limited to 2D cases. Some theoretical/analytical studies have been derived for convective instability of three-dimensional THC problems (Zhao et al. 2005, 2008a). In general, analytical and semi-analytical solutions for density-driven flow model are limited to steady-state conditions. 
Thus, the main goal of this study is to develop a 3D-transient semi-analytical solution for the problem of THC in a porous enclosure. Our main objective is to investigate an effective $3 \mathrm{D}$ configuration that cannot be simplified to $2 \mathrm{D}$. The motivations are i) to provide a reference solution that could be helpful for code benchmarking and ii) to understand the physical processes of THC in such a configuration (3D-tranisent). The semi-analytical solution is obtained using the FS method applied to the vector potential formulation of the governing equation, as in Shao et al. (2018). The extension to transient solutions represents an important technical feature of this work, as the applications of the FS method to density-driven problems have to date been limited to steady-state conditions.

\section{Problem and Model Statement}

\subsection{Problem description}

The problem under investigation is a saturated porous box which is commonly used as a benchmark for density-driven flow in porous media (Voss et al., 2010; Shao et al., 2018). The domain is a cubic box of size H, as shown in Figure 1a. All walls are assumed to be impermeable and adiabatic. Heat and mass fluxes only arise in the direction of imposed gradients of temperature and concentration. We consider a stable configuration of density-driven flow. Such a configuration is important in several applications and it is more relevant for benchmarking than unstable cases which can suffer from solution multiplicity (i.e. bifurcation and oscillations). As our goal is to investigate an effective 3D case, we impose horizontal-crossed thermal and solute gradients. Thus, we have a horizontal thermal gradient parallel to $\mathrm{x}$-direction and a horizontal solute gradient parallel to y-direction (Figure 1a). Constant temperatures are applied to the back and front walls of the domain and constant salinity concentrations are imposed on the left and right walls. With these boundary conditions, two circulation flows arise on two different planes 
and a 3D investigation becomes inevitable. Such a configuration can be found in several applications as in geothermal systems in coastal aquifers or in islands (e.g. GEOTREF project: https://geotref.com; van Lopik et al., 2015; Navelot et al., 2018; De Giorgio et al., 2018) where the sea/ocean can generate a salinity gradient and geothermal wells create a thermal gradient (Figure 1b). It is also important in applications involving variable density flow related to two different solute compositions in which the solute gradient can be horizontal and crossed. 
(a)

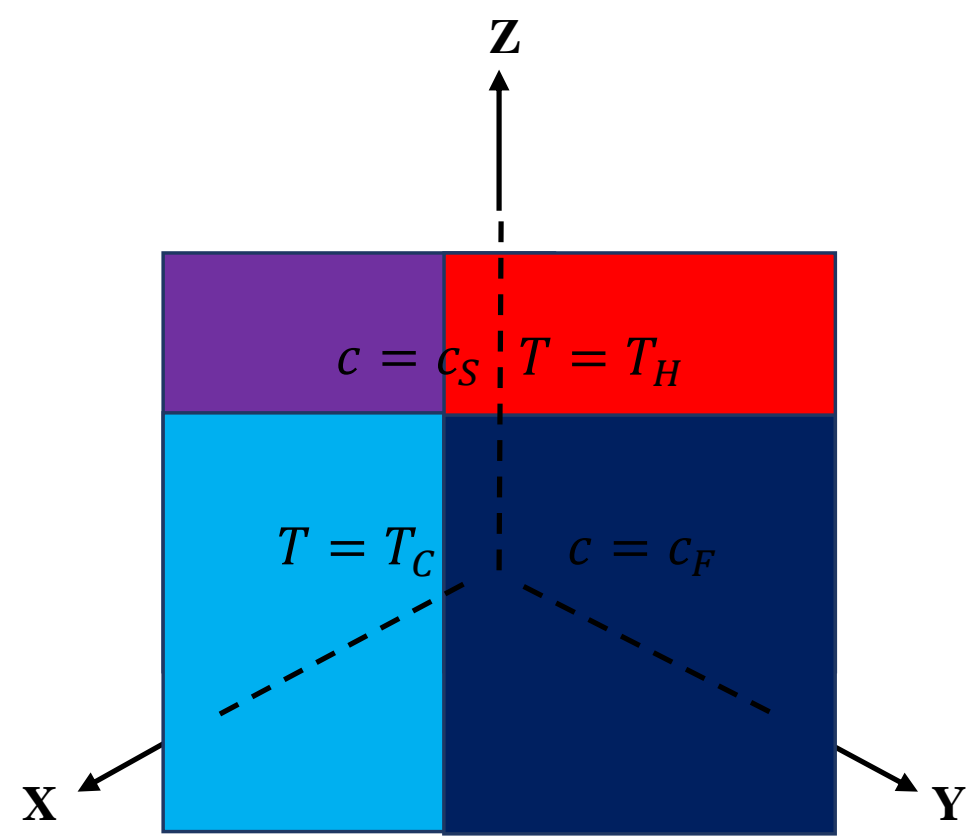

(b)

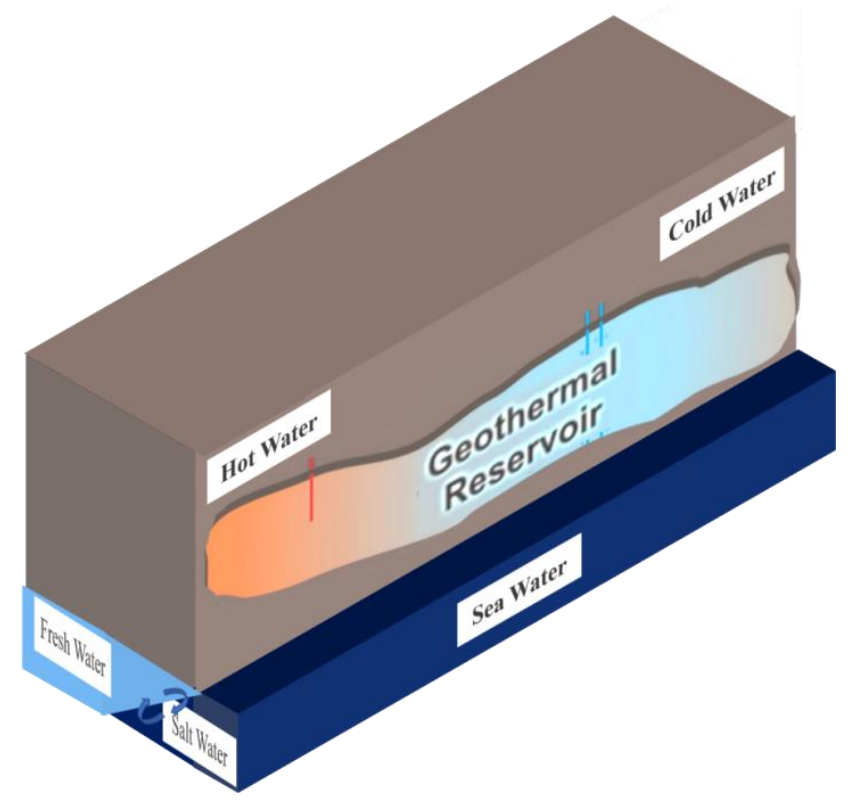

Figure 1. a) The conceptual problem of a cubic porous box with horizontal-crossed gradients of temperature and concentration and b) a conceptual model for a geothermal reservoir dealing with horizontal-crossed gradients of temperature and salinity.

\subsection{Model assumptions, governing equations and boundary and initial conditions}

We consider an isotropic and homogeneous porous medium and we assume local thermal equilibrium between the solid and liquid phases. The Soret and Dufour effects are neglected, as 
common in THC studies. These effects are unlikely to be significant in real systems (Vafai, 2015). The only process contributing to mixing is the molecular diffusion for solute transport and thermal conduction for heat transfer. Hydrodynamic dispersion processes are neglected. The viscosity dependence to the temperature is also neglected. Such an approximation could be valid for small range of temperature change. For the porous medium, the assumption of incompressibility leads to elimination of specific storage from the continuity equation. The flow, mass transport and heat transport equations have been written in the transient mode. Boussinesq approximation is considered. Thus, fluid density is assumed to be constant in all terms of the governing equations, except the buoyancy term. Under the above assumptions, the fluid flow equations consist of continuity equation and generalized Darcy's law in terms of equivalent fresh-water head:

$$
\begin{gathered}
\nabla \cdot \mathbf{q}=0 \\
\mathbf{q}=-\frac{\rho_{0} g k}{\mu}\left(\nabla h+\frac{\rho-\rho_{0}}{\rho_{0}} \mathbf{e}_{\mathbf{z}}\right)
\end{gathered}
$$

where $\mathbf{q}\left[L . T^{-1}\right]$ is the Darcy's velocity, $\rho_{0}\left[M . L^{-3}\right]$ is the fresh-water density at the reference temperature, $g\left[L . T^{-2}\right]$ is the Gravity acceleration, $k\left[L^{2}\right]$ is the permeability of the porous medium, $\mu\left[M \cdot L^{-1} \cdot T^{-1}\right]$ is the water viscosity, $h[L]$ is equivalent freshwater head, $\rho\left[M \cdot L^{-3}\right]$ is the density of salt water at a given temperature and $\mathbf{e}_{\mathbf{z}}$ is the upward vertical unit vector.

The governing equation for the saline mass transport is as follows:

$$
\varepsilon \frac{\partial c}{\partial t}+\mathbf{q} \cdot \nabla c-D_{m} \nabla \cdot \nabla c=0
$$


where $\varepsilon[-]$ is porosity, $c[-]$ is relative dimensionless concentration, $D_{m}\left[L^{2} \cdot T^{-1}\right]$ is the diffusion coefficient.

The heat transfer is governed by the equation of conservation of energy:

$$
\sigma \frac{\partial T}{\partial t}+\mathbf{q} \cdot \nabla T-\alpha \nabla \cdot \nabla T=0
$$

where, $\sigma[-]$ is the ratio of heat capacity, $T[\Theta]$ is the temperature, $\alpha\left[L^{2} \cdot T^{-1}\right]$ is the thermal diffusivity.

The flow and heat and mass transfer equations are coupled via the following linear mixture density equation:

$$
\rho=\rho_{0}\left(1+\beta_{C}\left(c-c_{0}\right)-\beta_{T}\left(T-T_{0}\right)\right)
$$

where, $\beta_{C}[-]$ and $\beta_{T}\left[\Theta^{-1}\right]$ are, respectively, the solute and thermal expansions, $c_{0}[-]$ is the reference relative salt concentration and $T_{0}[\Theta]$ is the reference temperature.

The boundary conditions are as follows:

$$
\begin{aligned}
& x=0 \rightarrow q_{x}=0 ; T=T_{H} ; \partial c / \partial x=0 \\
& x=H \rightarrow q_{x}=0 ; T=T_{C} ; \partial c / \partial x=0 \\
& y=0 \rightarrow q_{y}=0 ; c=c_{S} ; \partial T / \partial y=0 \\
& y=H \rightarrow q_{y}=0 ; c=c_{F} ; \partial T / \partial y=0 \\
& z=0 ; z=H \rightarrow q_{z}=0 ; \partial c / \partial z=0 ; \partial T / \partial z=0
\end{aligned}
$$

where $T_{H}[\Theta]$ and $T_{C}[\Theta]$ are the hot and cold temperatures, $c_{F}[-]$ is the relative salinity concentration of freshwater which is equal to zero and $c_{s}[-]$ is the relative salinity concentration 
of saltwater which is equal to one. For the transient cases, we assume that the initial temperature and concentration varies linearly with respect to $\mathrm{x}$ and $\mathrm{y}$, respectively.

It should be noted that existing extensive studies (Zhao et al., 2008b, 2016; Zhao, 2014) have demonstrated that chemical dissolution reactions can change both porosity and permeability of a porous medium and therefore affect the flow instability in the porous medium. This kind of instability is called the chemical dissolution-front instability and can interact with the THC flow (Zhao et al., 2013, 2015). However, since chemical reactions were neglected in this study, this issue was not considered, even though they should be considered in the future studies.

\section{The Fourier series solution}

The 3D semi-analytical solution is developed using the FS method also called Fourier-Galerkin method (Peyret, 2013). This method has been used to obtain semi-analytical solutions for several 2D density-driven flow problems (Henry, 1964; Segol, 1994, Simpson and Clement, 2003, 2004; Van Reeuwijk et al., 2009; Zidane et al., 2012, Younes and Fahs, 2014, 2015; Fahs et al., 2014, 2015, 2016; Shao et al. 2015, 2016; Koohbor et al., 2018). Shao et al. (2018) extended the FS method to solve a three-dimensional solute density-driven flow problem. In all the aforementioned works the FS method implementations have been limited to steady-state cases. This method has never been used to obtain transient solutions. In this work, we extend the steady-state FS method developed by Shao et al. (2018) to THC by including heat transfer processes and we develop a new implementation of this method to obtain transient solutions. In both cases (steady-state and transient), the solutions are obtained using the vector potential formulation of the governing equations. This formulation simplifies the resolution procedure by i) eliminating the pressure head which acts as a source term in the linear momentum conservation equation and may be at the origin of slower convergence of the Fourier series, ii) 
honoring the continuity equation and iii) ensuring homogeneous boundary conditions required for the FS method. The main steps of the FS method are recalled below:

\subsection{Vector potential formulation and non-dimensional system}

The continuity equation implies the existence of the vector potential $\left(\psi\left[L^{2} \cdot T^{-1}\right]\right)$ which is defined by (Guerrero-Martínez et al., 2017):

$$
\nabla \times \psi=\mathbf{q}
$$

The vector potential formulation of the governing equations can be obtained by applying the curl operator to Darcy's law and by substituting equation (7) into equations (3) and (4). The nondimensional form of the equations can be obtained using the following dimensionless variables:

$$
X=\frac{x}{H} ; Y=\frac{y}{H} ; Z=\frac{z}{H} ; \Psi=\frac{\Psi}{\alpha} ; \tau=\frac{t \alpha}{H^{2}} ; \theta=\frac{T-T_{C}}{T_{H}-T_{C}}
$$

Thus, by using the dimensionless variables and the vector potential and by assuming that the reference temperature is $T_{C}\left(T_{0}=T_{C}\right)$ and the reference concentration is $c_{F}\left(c_{0}=c_{F}\right)$, the governing equations become (more details can be found in Shao et al. (2018)):

$$
\begin{gathered}
\nabla^{2} \Psi_{X}-R a_{T}\left(N_{g} \frac{\partial c}{\partial Y}-\frac{\partial \theta}{\partial Y}\right)=0 \\
\nabla^{2} \Psi_{Y}+R a_{T}\left(N_{g} \frac{\partial c}{\partial X}-\frac{\partial \theta}{\partial X}\right)=0 \\
\nabla^{2} \Psi_{Z}=0 \\
\varepsilon \frac{\partial c}{\partial \tau}+(\nabla \times \Psi) \nabla c-\frac{1}{L e} \nabla^{2} c=0 \\
\sigma \frac{\partial \theta}{\partial \tau}+(\nabla \times \Psi) \nabla \theta-\nabla^{2} \theta=0
\end{gathered}
$$


where $\Psi_{X}, \Psi_{Y}, \Psi_{Z}$ are the components of the vector potential $\Psi, R a_{T}=\frac{\rho_{0} g k H \beta_{T}\left(T_{H}-T_{C}\right)}{\mu \alpha}$ is the thermal Rayleigh number representing the ratio of the thermal buoyancy to thermal diffusivity, $N_{g}=\frac{\beta_{C}\left(c_{S}-c_{F}\right)}{\beta_{T}\left(T_{H}-T_{C}\right)}$ is the Gravity number which is the ratio of mass buoyancy to thermal buoyancy and $L e=\frac{\alpha}{D_{m}}$ is the Lewis number expressing the ratio of thermal diffusivity to mass diffusivity.

\subsection{Homogeneous boundary conditions}

The FS method requires homogeneous boundary conditions to ensure periodicity. For the vector potential, the impermeable boundary conditions can be expressed as follows (for more details readers can refer to Shao et al. 2018):

$$
\begin{array}{ll}
\partial \Psi_{X} / \partial X=\Psi_{Y}=\Psi_{Z}=0, & \text { at } X=0,1 \\
\partial \Psi_{Y} / \partial Y=\Psi_{X}=\Psi_{Z}=0, & \text { at } \mathrm{Y}=0,1 \\
\partial \Psi_{Z} / \partial Z=\Psi_{X}=\Psi_{Y}=0, & \text { at } \mathrm{Z}=0,1
\end{array}
$$

Thus, the flow boundary conditions are homogeneous. The solute and thermal boundary conditions are homogeneous except in the $\mathrm{x}$ and $\mathrm{y}$ directions, respectively. To get homogeneous boundary conditions, we use the following shifted concentration and temperature:

$$
\begin{aligned}
& C=c+(Y-1) \\
& \Theta=\theta+(X-1)
\end{aligned}
$$

The final system of equations become:

$$
\nabla^{2} \Psi_{X}-R a_{T}\left(N_{g} \frac{\partial C}{\partial Y}-N_{g}-\frac{\partial \Theta}{\partial Y}\right)=0
$$




$$
\begin{gathered}
\nabla^{2} \Psi_{Y}+R a_{T}\left(N_{g} \frac{\partial C}{\partial X}-\frac{\partial \Theta}{\partial X}+1\right)=0 \\
\nabla^{2} \Psi_{Z}=0 \\
\varepsilon \frac{\partial C}{\partial \tau}-\frac{\partial \Psi_{Y}}{\partial Z} \frac{\partial C}{\partial X}+\frac{\partial \Psi_{X}}{\partial Z} \frac{\partial C}{\partial Y}+\left(\frac{\partial \Psi_{Y}}{\partial X}-\frac{\partial \Psi_{X}}{\partial Y}\right) \frac{\partial C}{\partial Z}-\frac{1}{L e}\left(\frac{\partial^{2} C}{\partial X^{2}}+\frac{\partial^{2} C}{\partial Y^{2}}+\frac{\partial^{2} C}{\partial Z^{2}}\right)-\frac{\partial \Psi_{X}}{\partial Z}=0 \\
\sigma \frac{\partial \Theta}{\partial \tau}-\frac{\partial \Psi_{Y}}{\partial Z} \frac{\partial \Theta}{\partial X}+\frac{\partial \Psi_{X}}{\partial Z} \frac{\partial \Theta}{\partial Y}+\left(\frac{\partial \Psi_{Y}}{\partial X}-\frac{\partial \Psi_{X}}{\partial Y}\right) \frac{\partial \Theta}{\partial Z}-\left(\frac{\partial^{2} \Theta}{\partial X^{2}}+\frac{\partial^{2} \Theta}{\partial Y^{2}}+\frac{\partial^{2} \Theta}{\partial Z^{2}}\right)+\frac{\partial \Psi_{Y}}{\partial Z}=0
\end{gathered}
$$

The third component of the flow system (equation (19)) associated to the boundary conditions leads to $\Psi_{Z}=0$. Thus, equation (19) and $\Psi_{Z}$ can be eliminated from the final system.

\subsection{The spectral system}

As boundary conditions are homogeneous, the components of the vector potential $\left(\Psi_{X}, \Psi_{y}\right)$, shifted concentration $(C)$ and shifted temperature $(\Theta)$ can be expressed as Fourier series in the spectral space. The Fourier series that satisfy the boundary conditions are as follows:

$$
\begin{aligned}
& \Psi_{x}(X, Y, Z)=\sum_{i=0}^{N i} \sum_{j=1}^{N j} \sum_{k=1}^{N k} A_{i, j, k} \cos (i \pi X) \sin (j \pi Y) \sin (k \pi Z) \\
& \Psi_{y}(X, Y, Z)=\sum_{l=1}^{N l} \sum_{m=0}^{N m} \sum_{n=1}^{N n} B_{l, m, n} \sin (l \pi X) \cos (m \pi Y) \sin (n \pi Z) \\
& C(X, Y, Z)=\sum_{u=0}^{N u} \sum_{v=1}^{N v} \sum_{w=0}^{N w} E_{u, v, w} \cos (u \pi X) \sin (v \pi Y) \cos (w \pi Z) \\
& \Theta(X, Y, Z)=\sum_{s=1}^{N s} \sum_{p=0}^{N p} \sum_{t=0}^{N t} G_{s, p, t} \sin (s \pi X) \cos (p \pi Y) \cos (t \pi Z)
\end{aligned}
$$

where $A_{i, j, k}, B_{l, m, n}, E_{u, v, w}$ and $G_{s, p, t}$ are the Fourier series coefficients, $N i, N j, N k, N l, N m$, $N n, N u, N v, N w, N s, N p, N t$ are the truncation orders of the Fourier series in the space directions. 
The Fourier series coefficient can be calculated by substituting the Fourier series into equations (17) -(18) -(20) and (21) and by projecting the resulting equation into the spectral space using the Fourier modes as trial functions. The final spectral system can be written as follows:

$$
\begin{aligned}
& R F X_{I, J, K}=-\pi^{2}\left(I^{2}+\alpha_{I} J^{2}+\alpha_{I} K^{2}\right) A_{I, J, K}-\frac{N_{g} R a_{T} \alpha_{I}}{\pi} \sum_{v=1}^{N v} \sum_{w=0}^{N w} v E_{I, v, w}^{\prime} \Gamma_{J, v} \Gamma_{K, w} \\
& +\frac{2 N_{g} R a_{T}}{\pi^{2}} \Gamma_{J, 0} \Gamma_{K, 0} \delta_{I, 0}-\frac{R a_{T} J}{\pi} \sum_{s=1}^{N s} \sum_{t=0}^{N t} G_{s, J, t}^{\prime} \Gamma_{s, I} \Gamma_{K, t} \\
& (I=0, \ldots, N i ; J=1, \ldots, N j ; K=1, \ldots, N k) \\
& R F Y_{L, M, N}=-\pi^{2}\left(\alpha_{M} L^{2}+M^{2}+\alpha_{M} N^{2}\right) B_{L, M, N}-\frac{L N_{g} R a_{T}}{\pi} \sum_{v=1}^{N v} \sum_{w=0}^{N w} E_{L, v, w}^{\prime} \Gamma_{v, M} \Gamma_{N, w} \\
& -\frac{R a_{T} \alpha_{M}}{\pi} \sum_{s=1}^{N s} \sum_{t=0}^{N t} s G_{s, M, t}^{\prime} \Gamma_{L, s} \Gamma_{N, t}+\frac{2 R a_{T}}{\pi^{2}} \Gamma_{L, 0} \Gamma_{N, 0} \delta_{M, 0} \\
& (L=1, \ldots, N l ; M=0, \ldots, N m ; \mathrm{N}=1, \ldots, N n) \\
& R T_{U, V, W}=\varepsilon \alpha_{U} \alpha_{W} \frac{d E_{\tau, U, V, W}}{d \tau} \\
& +\frac{\pi^{2}}{8} \sum_{u=0}^{N u} \sum_{v=1}^{N v} \sum_{w=0}^{N w} E_{u, v, w}\left[\sum_{i=0}^{N i} \sum_{j=1}^{N j} \sum_{k=1}^{N k} A_{i, j, k} \xi_{S, i, s}\left(k v \gamma_{V, j, v} \eta_{W, k, w}+j w \kappa_{V, j, v} \zeta_{W, k, w}\right)\right. \\
& \left.+\sum_{l=1}^{N l} \sum_{m=0}^{N m} \sum_{n=1}^{N n} B_{l, m, n} \kappa_{V, j, v}\left(n u \zeta_{U, l, u} \eta_{W, n, w}-w l \eta_{U, l, u} \zeta_{W, n, w}\right)\right] \\
& +\frac{\pi^{2}}{L e}\left(U^{2} \alpha_{W}+V^{2} \alpha_{U} \alpha_{W}+W^{2} \alpha_{U}\right) E_{U, V, W}-\pi W \alpha_{U} A_{U, V, W}^{\prime} \\
& (U=0, \ldots, N u ; V=1, \ldots, N v ; W=0, \ldots, N w) \\
& R E_{S, P, T}=\sigma \alpha_{P} \alpha_{T} \frac{d G_{\tau, S, P, T}}{d \tau} \\
& +\frac{\pi^{2}}{8} \sum_{s=1}^{N s} \sum_{p=0}^{N p} \sum_{t=0}^{N t} G_{s, p, t}\left[\sum_{i=0}^{N i} \sum_{j=1}^{N i} \sum_{k=1}^{N k} A_{i, j, k} \kappa_{S, i, s}\left(j t \eta_{P, j, p} \zeta_{T, k, t}-k p \zeta_{P, p, j} \eta_{T, k, t}\right)\right. \\
& \left.-\sum_{l=1}^{N l} \sum_{m=0}^{N m} \sum_{n=1}^{N n} B_{l, m, n} \eta_{P, m, p}\left(l t \kappa_{S, l, s} \zeta_{T, n, t}+n s \gamma_{S, l, s} \eta_{T, n, t}\right)\right] \\
& +\pi^{2}\left(S^{2} \alpha_{P} \alpha_{T}+P^{2} \alpha_{T}+T^{2} \alpha_{P}\right) G_{S, P, T}+\pi T \alpha_{P} B_{S, P, T}^{\prime} \\
& (S=1, \ldots, N s ; P=0, \ldots, N p ; \mathrm{T}=0, \ldots, N t)
\end{aligned}
$$


Where, $R F X, R F Y, R T$ and $R E$ are the residuals corresponding to the flow and mass and heat transport equations, respectively. The coefficients of equations (25)-(29) are given in Appendix A.

The spectral system (equations (25)-(29)) is a system of differential algebraic equations that contains a set of ordinary differential equations (mass and heat transport residuals) and algebraic nonlinear equations (residuals of the $\mathrm{x}$ and $\mathrm{y}$-components of the flow equations). The steady-state spectral system can be obtained by dropping out the transient terms (first terms) in equations (28) and (29) which yields an algebraic nonlinear system.

\subsection{Solving the spectral system}

Solving the spectral system (equations (25)-(29)) is a crucial step to obtain the Fourier coefficients and the semi-analytical solution. For both transient and steady-state configurations, the performance of the FS method depends on the way in which the spectral system is solved. This is a challenging task as, for sharp solutions (i.e. high Rayleigh or Lewis numbers), the FS method requires large number of Fourier modes to avoid the Gibbs phenomenon (Peyert, 2013) for which the solution could become impractical due to the computational cost and nonlinearity. This would undermine the first advantage of the semi-analytical solution regarding its practicality when compared against numerical solutions. Furthermore, for transient configurations, the accuracy of the solution depends on the numerical technique used for time integration. Here, we present the numerical implementations used to ease these challenges and to obtain accurate solutions in tractable CPU time. 


\section{- Transient solution}

As shown in the previous section, in the spectral space, the problem of THC in a porous cubic box is modeled as a system of differential algebraic equations (DAEs) that contains a set of ordinary differential equations (ODEs) (equations 28 and 29) and algebraic nonlinear constraints (equations 26 and 27). The unknowns are the Fourier series coefficients $(A, B, E$ and $G)$. To obtain an accurate and efficient solution, we convert the DAEs system into an ODEs system. To do so, we use equations (26) and (27) to analytically express the coefficients $A$ and $B$ as function of $E$ and $G$ (see Appendix B). We then substitute the analytical expressions of $A$ and $B$ into equations (28) and (29). This procedure reduces, on one hand, the number of unknowns as the new system can be solved with only the Fourier series coefficients of the concentration and temperature as primary unknowns (i.e. $E$ and $G$ ), and on the other hand, simplifies and improves the resolution procedure, as it is well-known that ODEs systems are more stable than DEAs systems. For stable and accurate time integration of the resulting ODEs system with strong nonlinearity, we use the sophisticated and mature time integration solver DASPK (https://techtransfer.universityofcalifornia.edu/NCD/10326.html). This solver provides high accuracy as it is based on the Backward Difference Formulas (BDF) which is an implicit high order integration technique ( $\mathrm{Li}$ and Petzold, 1999). The solver adapts both the time step size and time integration order (up to fifth order) to reach high accuracy and stable solutions. Higher order time integration method also improves the efficiency of the integration procedure as it allows for a large time step size. The Fixed Leading Coefficient Backward Difference Formulas (FLCBDF) is used in DASPK to deal with variable time step size and integration order. With this method the system is converted, at each time step, to a system of nonlinear algebraic equations. In DASPK, this system is solved using the modified Newton's method (Brown et al., 1994). The 
Jacobian matrix can be evaluated numerically (i.e. using finite difference approximation) or provided by the user. The assembly of the numerical Jacobian is computationally consuming, as the system is fully-dense (i.e. most of the elements of the Jacobian matrix are nonzero). Thus, in our implementation, we provide the Jacobian matrix analytically in order to improve the solution performance. The resulting linear system can be solved using either direct or iterative methods. We choose the iterative method (Krylov method) to gain computational efficiency owing to the highly dense nature of the matrices involved and due to the system high-dimensionality in 3D (Brown et al. 1994). Our choice for an iterative method is also justified by the memory limitation with direct methods for large linear systems. Both relative and absolute local error tolerances are prescribed to be $10^{-8}$.

\section{- Steady-state solution}

The spectral steady-state system can be obtained by dropping out the transient terms (first terms) in equations (28) and (29). This yields an algebraic nonlinear system with the Fourier coefficients as unknowns. To solve this system, we extend the implementation developed by Shao et al. (2018) to deal with THC. Thus, we first reduce the number of unknowns by expressing the Fourier coefficients $A$ and $B$ as function of $E$ and $G$, as in the transient solution (see appendix B). The resulting nonlinear system is then solved using the nonlinear solver of the IMSL library (http://www.roguewave.com/products-services/imsl-numerical-libraries). To improve the solver performance, we provide the analytical Jacobian matrix.

In both transient and steady-state solutions, the nonlinear solvers require the evaluation of the residual vector. The evaluation of this vector involves six nested summations in the convection terms. Thus, when a large number of Fourier coefficients should be used to avoid the Gibbs phenomenon, the solution becomes computationally impractical. As in Shao et al. (2018), we use 
the properties of the Kronecker delta function to reduce the number of nested summations to three.

\section{Results and Discussion: Verification and benchmarking}

Three targets are discussed in this section: i) as two numerical codes have been developed to solve the steady-state and transient spectral systems, we verify the correctness of these codes by comparison with finite element solutions obtained using a commercial code (COMSOL Multiphysics $\left.{ }^{\circledR}\right)$, ii) comparison against standard finite element solutions, for complex cases involving sharp concentration and/or temperature distributions, are also used to examine the worthiness of the developed semi-analytical solution in benchmarking numerical codes and iii) we use the developed solution to provide new physical insights on the three-dimensional THC processes in the case of crossed-horizontal gradients, under both steady-state and transient conditions. In our analysis, as common in the literature, we use the average Nusselt $(\overline{\mathrm{Nu}})$ and Sherwood $(\overline{S h})$ numbers to characterize the rates of heat and mass transfer to the domain, respectively. Both steady-state values and time-variations of these numbers are investigated to assess steady-state and transient solutions, respectively. $\overline{N u}$ and $\overline{S h}$ are calculated using the Fourier series as follows:

$$
\begin{aligned}
& \overline{S h}=\left.\int_{0}^{1} \int_{0}^{1} \frac{\partial c}{\partial Y}\right|_{Y=0} d X d Z=\pi \sum_{v=1}^{N v} v E_{0, v, 0}-1 \\
& \overline{N u}=\left.\int_{0}^{1} \int_{0}^{1} \frac{\partial \theta}{\partial X}\right|_{X=0} d Y d Z=\pi \sum_{s=1}^{N s} s G_{s, 0,0}-1
\end{aligned}
$$




\subsection{Verifications}

To gain confidence in the correctness of the codes developed to solve the steady-state and transient spectral systems of the semi-analytical solution, we compare their results with a finite element solution (FE) obtained using COMSOL Multiphysics ${ }^{\circledR}$. The steady-state semi-analytical solution is denoted by 'SA-steady' and the transient solution is termed as 'SA-transient'. To avoid numerical artifacts in the finite element solution, that could lead to discrepancy with the semi-analytical solution, we consider relatively simple cases dealing with smooth temperature and concentration distributions (low convective flow regime). Thus, we examine two cases dealing with $R a_{T}=10, N_{g}=1.5$ and $L e=2$ (denoted by 'test case 1') and $R a_{T}=100, N_{g}=1$ and $L e=0.5$ (denoted by 'test case 2'), respectively. In 'test case 1' the conduction-diffusion regime is dominating (low thermal Rayleigh number), the mass transport is less diffusive than heat transfer $(L e>1)$ and the convective flow is solute-dominated $\left(N_{g}>1\right)$. In the 'test case 2', the convective flow is more pronounced than 'test case 1' (higher thermal Rayleigh number), molecular diffusion is more intense than thermal conduction $(L e<1)$ and thermal and solute convective flows occur in equal proportions $\left(N_{g}=1\right) . L e<1$ is not common in THC because heat is more diffusive than mass transfer, but such a configuration can be found in DDC. For the transient solutions, in both test cases, we consider $\varepsilon=0.1$ (porosity) and $\sigma=0.46$ (specific heat ratio).

The FS method could suffer from Gibbs oscillations around discontinuities. Thus, appropriate number of Fourier modes should be used to obtain stable solutions. To do so, we use the technique developed by Fahs et al. (2014) that proceeds by increasing progressively the number of Fourier modes until reaching stable values of $\overline{N u}$ and $\overline{S h}$. In each space direction, we use the 
same number of Fourier modes for all variables $\left(\Psi_{x}, \Psi_{y}, c\right.$ and $\left.\Theta\right)$. Thus, we have $N X=N i=N l=N u=N s, \quad N Y=N j=N m=N v=N p \quad$ and $\quad N Z=N k=N n=N w=N t$. For the 'test case 1', stable solutions (both SA-steady and SA-transient) have been obtained with $N X=3, N Y=12$ and $N Z=6$. In 'test case 2', the temperature and concentration distributions are sharper than 'test case 1'. Thus, more Fourier modes are required to get a stable solution which is obtained with $N X=3, N Y=24$ and $N Z=20$. It should be mentioned that stable isotherms and concentration contours can be obtained with smaller number of Fourier modes, but these numbers were required to get stable $\overline{N u}$ and $\overline{S h}$.

The COMSOL model has been built by coupling three modules: 'Darcy's Law - dl', 'Heat Transfer in Porous Media $-h t$ ' and 'Transport of Diluted Species in Porous media $-t d s$ '. The density is assumed to be a function of temperature and concentration as in equation (5). The Boussinesq approximation is implemented in COMSOL by assuming constant density in the three modules (' $d l$ ', ' $h t$ ' and ' $t d s$ ') and including variable density in the Gravity term. The physical parameters used in COMSOL to simulate 'test case 1' and 'test case 2' are given in Table 1. Transient simulations are performed in COMSOL. This is useful to avoid convergence issues usually encountered with steady-state solutions. It is also helpful for the comparison against the SA-transient solution. Steady-state solutions are obtained with COMSOL by letting the transient solutions evolve until a permanent regime is obtained. For each case, a grid convergence analysis is performed to obtain a mesh-independent solution. $\overline{N u}$ and $\overline{S h}$ are used as metrics for the convergence analysis. 3D triangular grids, generated by the COMSOL meshing tool (physical controlled mesh), are used for the space domain discretization. For 'test case 1' and 'test case 2', mesh-independent solutions are obtained using grids consisting of about $35 \mathrm{~K}$ and $100 \mathrm{~K}$ elements, respectively. 
Table 1. Non-dimensional parameters used in the semi-analytical solutions and physical parameters used in COMSOL for different test cases.

\begin{tabular}{|c|c|c|c|c|}
\hline \multicolumn{5}{|c|}{ Non-dimensional parameters used in the semi-analytical solutions } \\
\hline & $R a_{T}$ & $N_{g}$ & Le & $\sigma$ \\
\hline 'test case 1 ' & 10 & 1.5 & 2.0 & 0.46 \\
\hline 'test case 2 ' & 100 & 1.0 & 0.5 & 0.46 \\
\hline 'test case 3' & 100 & 1.5 & 2.0 & 0.46 \\
\hline \multicolumn{5}{|c|}{ Invariable physical parameters used in COMSOL } \\
\hline \multirow{3}{*}{\multicolumn{3}{|c|}{$\begin{array}{l}\text { Porous box side } \\
\text { Porosity } \\
\text { Freshwater density }\end{array}$}} & \multirow{2}{*}{\multicolumn{2}{|c|}{$\begin{array}{c}H=1.0 \mathrm{~m} \\
e=0.1\end{array}$}} \\
\hline & & & & \\
\hline & & & \multicolumn{2}{|c|}{$\rho_{0}=1000 \mathrm{~kg} \cdot \mathrm{m}^{-3}$} \\
\hline \multicolumn{3}{|c|}{ Solid phase density } & \multicolumn{2}{|c|}{$\rho_{s}=2000 \mathrm{~kg} \cdot \mathrm{m}^{-3}$} \\
\hline \multicolumn{3}{|l|}{ Gravity } & \multicolumn{2}{|c|}{$g=9.8 \mathrm{~m} \cdot \mathrm{s}^{-2}$} \\
\hline \multicolumn{3}{|l|}{ Viscosity } & \multicolumn{2}{|c|}{$\mu=10^{-3} \mathrm{~kg} \cdot \mathrm{m}^{-1} \cdot \mathrm{s}^{-1}$} \\
\hline \multicolumn{3}{|c|}{ Cold temperature } & \multicolumn{2}{|c|}{$T_{C}=273.15 \mathrm{~K}$} \\
\hline \multicolumn{3}{|c|}{ Hot temperature } & \multicolumn{2}{|c|}{$T_{H}=274.15 \mathrm{~K}$} \\
\hline \multicolumn{3}{|c|}{ Concentration of saltwater } & \multicolumn{2}{|c|}{$c_{S}=1 \mathrm{~mol} \cdot \mathrm{m}^{-3}$} \\
\hline \multicolumn{3}{|c|}{ Concentration of freshwater } & \multicolumn{2}{|c|}{$c_{F}=0$ mol. $\mathrm{m}^{-3}$} \\
\hline \multicolumn{3}{|c|}{ Thermal expansion coefficient of water } & \multicolumn{2}{|c|}{$\beta_{T}=10^{-2} \mathrm{~K}^{-1}$} \\
\hline \multicolumn{3}{|c|}{ Thermal capacity of Water } & \multicolumn{2}{|c|}{$c p_{f}=4200 \mathrm{~J} \cdot \mathrm{kg}^{-1} \cdot \mathrm{K}^{-1}$} \\
\hline \multicolumn{3}{|c|}{ Thermal capacity of soil } & \multicolumn{2}{|c|}{$c p_{s}=850 \mathrm{~J} \cdot \mathrm{kg}^{-1} \cdot \mathrm{K}^{-1}$} \\
\hline \multicolumn{3}{|c|}{ Thermal Conductivity of Water } & \multicolumn{2}{|c|}{$\lambda_{f}=0.65 \mathrm{~W} \cdot \mathrm{m}^{-1} \cdot \mathrm{K}^{-1}$} \\
\hline \multicolumn{3}{|c|}{ Thermal Conductivity of Soil } & \multicolumn{2}{|c|}{$\lambda_{s}=1.59 \mathrm{~W} \cdot \mathrm{m}^{-1} \cdot \mathrm{K}^{-1}$} \\
\hline \multicolumn{5}{|c|}{ Variable physical parameters used in COMSOL } \\
\hline & $\begin{array}{l}\text { Permeability } \\
K\left(\mathrm{~m}^{2}\right)\end{array}$ & \multicolumn{2}{|c|}{$\begin{array}{c}\text { Molecular Diffusion } \\
D_{m}\left(\mathrm{~m}^{2} \cdot \mathrm{s}^{-1}\right) \\
\end{array}$} & $\begin{array}{c}\text { Mass Expansion } \\
\beta_{C}\left(\mathrm{~m}^{3} \cdot \mathrm{mol}^{-1}\right)\end{array}$ \\
\hline 'test case 1' & $3.634 \times 10^{-11}$ & \multicolumn{2}{|c|}{$1.78 \times 10^{-7}$} & \\
\hline 'test case 2 ' & $3.634 \times 10^{-10}$ & & & \\
\hline 'test case 3 ' & $3.634 \times 10^{-10}$ & & & \\
\hline
\end{tabular}

$* \varepsilon$ and $\sigma$ are only used for transient solutions. 

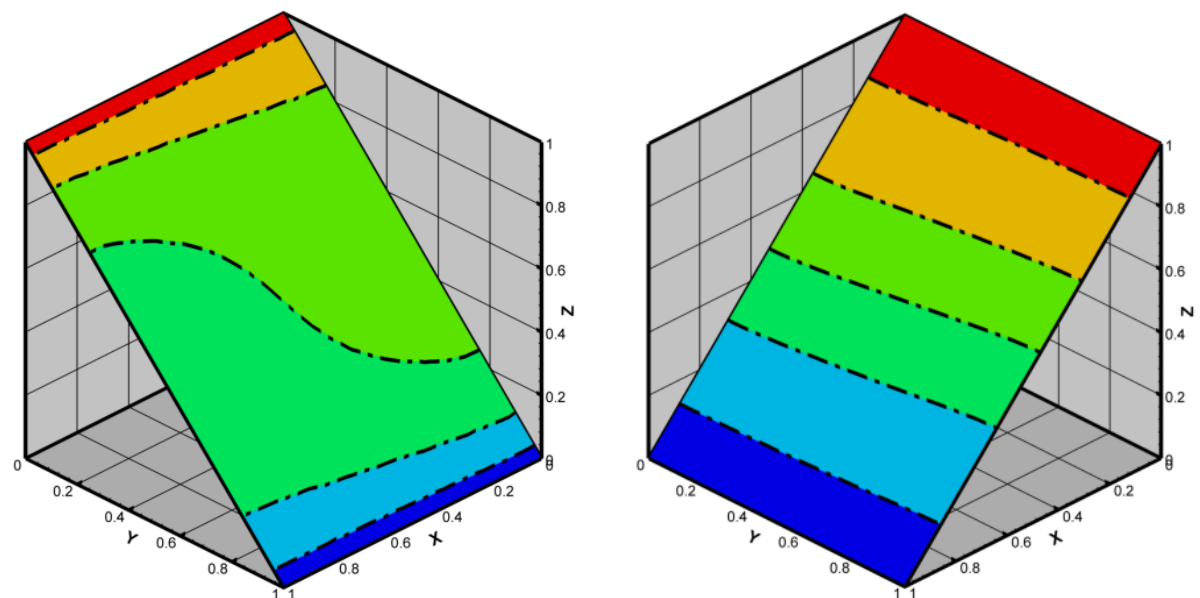

'Test case 1': $R a_{T}=10, N_{g}=1.5$ and $L e=2$
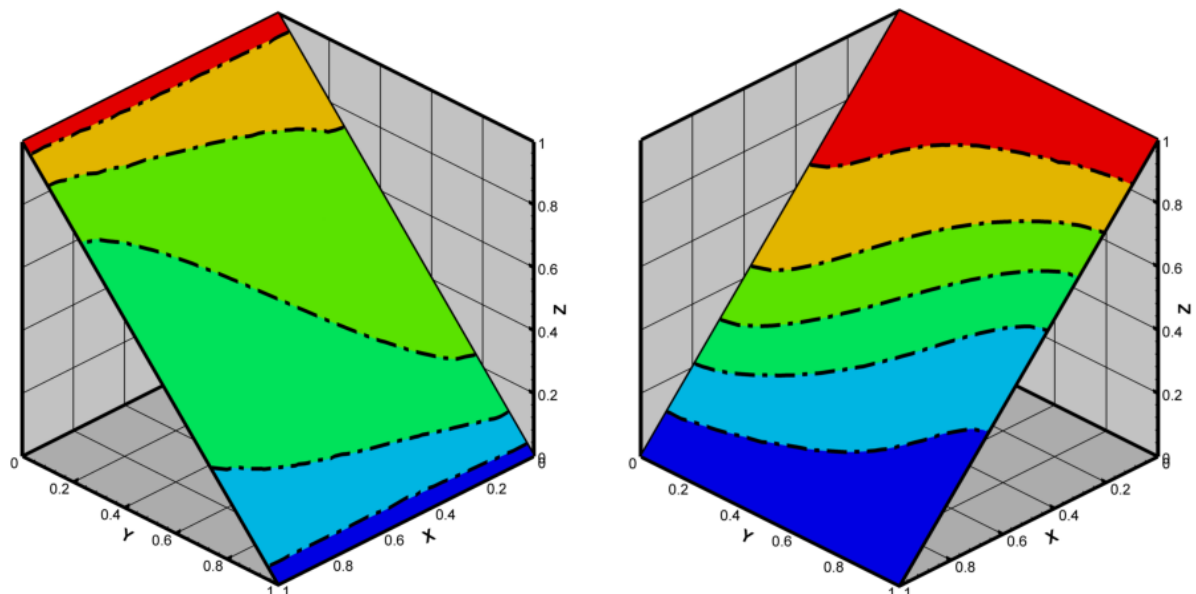

'Test case 2': $R a_{T}=100, N_{g}=1$ and $L e=0.5$

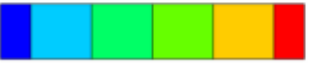

0.10 .30 .50 .70 .9

Figure 2. Comparison of the steady-state semi-analytical solution (Flood map) and COMSOL (dashed lines): concentration contours (left) and isotherms (right) in two diagonal plans.

Figure 2 exemplifies results of the comparison between the 'SA-steady' solution against COMSOL. It shows the main concentration contours and isotherms on the diagonal plans along the solute and thermal gradients, respectively. These plans are helpful to understand the effects of thermal and solute gradients on concentration and temperature distributions. For 'test case 1', the figure shows a clear three-dimensional structure of the concentration distribution while the temperature field is almost two-dimensional because a conduction regime is dominating (low $R a_{T}$ and $L e>1$ ). For higher thermal Rayleigh number, as in 'test case 2', both the concentration 
distribution and temperature field have three-dimensional structures. Similar plots are made in Figure 3 for the comparison between the 'SA-transient' solution and COMSOL, at $\tau=0.005$ for 'test case 1' and $\tau=0.001$ for 'test case 2'. At $\tau=0.005$, the concentration contours and isotherms are almost linear in 'test case 1'. Thus, the convective flow is relatively weak. The convective flow is more pronounced at earlier time in 'test case 2'. We also compare the 'SAsteady' solutions and COMSOL based on $\overline{N u}$ and $\overline{S h}$. The corresponding values are given in Table 2. For the SA-transient solutions, we plot, in Figure 4, the time variation of $\overline{N u}$ and $\overline{S h}$. This figure indicates that, for 'test case 1', mass transfer to the domain increases with time while the heat transfer flux is almost constant. For 'test case 2', two variation regimes can be distinguished. Both $\overline{N u}$ and $\overline{S h}$ increase with time at the beginning until reaching their maximal values. After a critical time, they decrease and reach asymptotic values. Figures 2, 3 and 4 and Table 2 show excellent agreement between the results of the semi-analytical solutions and COMSOL. Moreover, the asymptotic values of transient $\overline{N u}$ and $\overline{S h}$ are equal to the corresponding steady-state values, as in Table 2. These results provide compelling evidence in confirming the correctness of the codes developed to solve the steady-state and transient spectral systems of the semi-analytical solution. It should be mentioned that relatively fine levels of grids have been used to obtain independent-mesh values of $\overline{N u}$ and $\overline{S h}$, but in general stable isotherms and concentration contours can be obtained with coarser grids. 

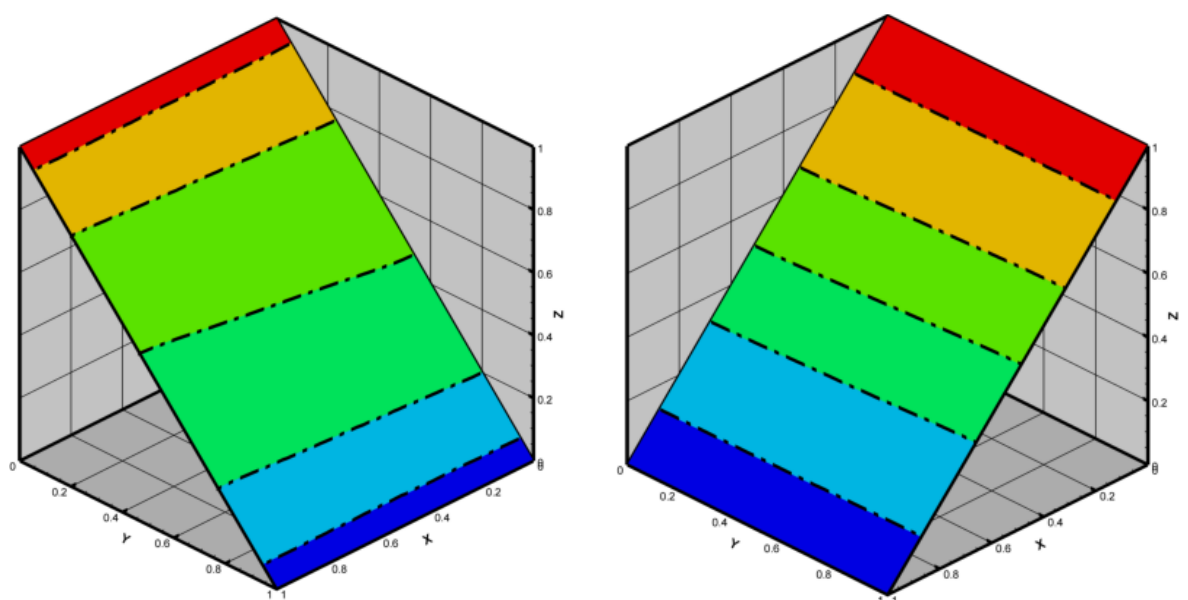

'Test case 1' $\left(R a_{T}=10, N_{g}=1.5\right.$ and $\left.L e=2\right)$ at $\tau=0.005$
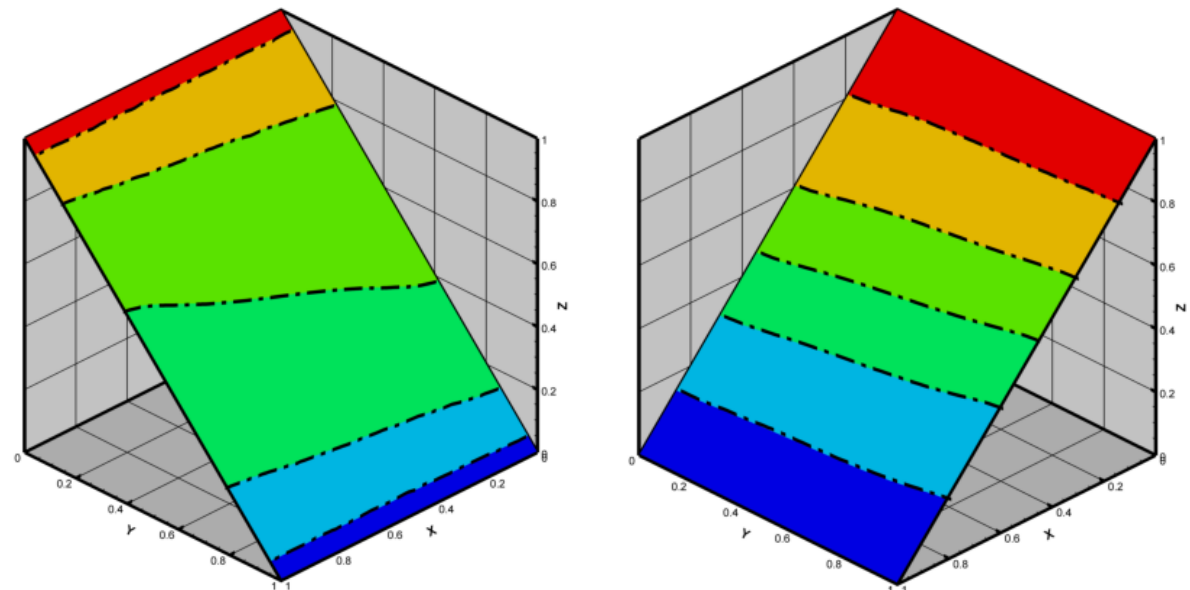

'Test case 2' $\left(R a_{T}=100, N_{g}=1\right.$ and $\left.L e=0.5\right)$ at $\tau=0.001$

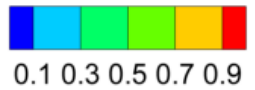

Figure 3. Comparison of the transient semi-analytical solution (Flood map) and COMSOL (dashed lines): concentration contours (left) and isotherms (right) in two diagonal plans ( $\tau$ is the non-dimensional time, $\varepsilon=0.1$ and $\sigma=0.46$ ). 
Table 2. Average Nusselt $(\overline{N u})$ and Sherwood $(\overline{S h})$ numbers for different test cases obtained using the steady-state semi-analytical solution (SA-steady) and COMSOL. COMSOL-v5.3 is used to denote a previous version of the software while COMSOL is used for the newer release (v5.4). All parameters for the three test cases are given in Table 1.

\begin{tabular}{|c|c|c|c|}
\hline Test case & Method & $\overline{S h}$ & $\overline{N u}$ \\
\hline \multirow{2}{*}{ 'test case 1' } & SA-steady & 1.44 & 1.04 \\
\cline { 2 - 4 } & COMSOL & 1.42 & 1.04 \\
\hline \multirow{2}{*}{ 'test case 2' } & SA-steady & 1.37 & 2.19 \\
\cline { 2 - 4 } & COMSOL & 1.37 & 2.16 \\
\hline \multirow{3}{*}{ 'test case 3' } & SA-steady & 5.17 & 1.25 \\
\cline { 2 - 4 } & COMSOL & 5.01 & 1.25 \\
\cline { 2 - 4 } & COMSOL-v5.3 & 1.97 & 1.17 \\
\hline
\end{tabular}
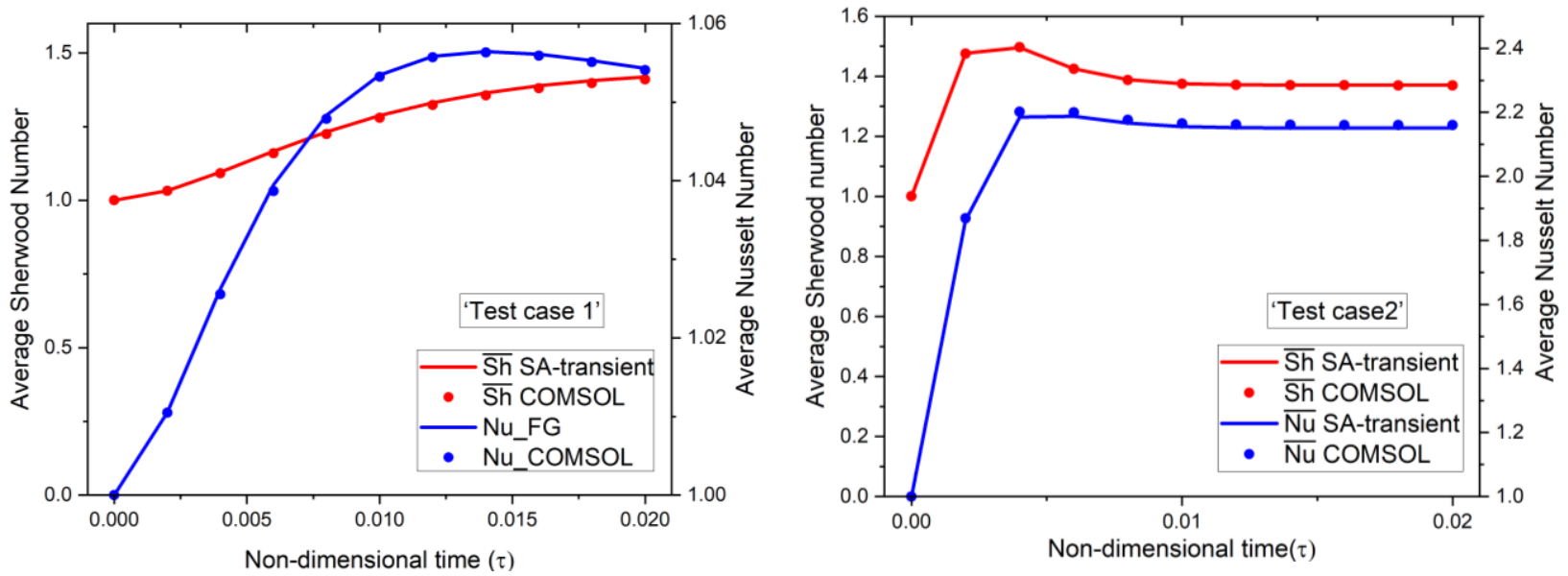

Figure 4. Time variation of the average Nusselt $(\overline{N u})$ and Sherwood $(\overline{S h})$ numbers: Comparison of the transient Fourier series solution (SA-transient) and COMSOL for 'test case 1' and 'test case 2'. All parameters for both test cases are given in Table 1.

\subsection{Benchmarking}

We consider a complex case dealing with sharper temperature and concentration distributions than the previous test cases. To do so, we assume high values for $R a_{T}(=100), N_{g}(=1.5)$ and Le $(=2)$. The new test case is called 'test case 3'. Its non-dimensional and physical parameters are given in Table 1. The semi-analytical solution is obtained with: $N X=17, N Y=18$ and $N Z=15$. Our first simulations have been performed using COMSOL version (5.3). The simulations are performed using a grid of about $100 \mathrm{~K}$ elements, as in 'test case 2'. COMSOL is bound to run into convergence difficulties and cannot reach the permanent regime. Incoherent 
results can be observed with negative temperatures and concentrations and larger values beyond physics (i.e. negative temperatures and concentrations or values largely greater than 1). Convergence issues and unphysical values of concentration and temperature are related to spurious oscillations because 'test case 3 ' is a convection-dominated problem for which the finite element method, used in COMSOL, can lead to instabilities. It is well-known that these oscillations can be removed by using a grid respecting a Péclet number less than 1 in the whole computational domain. In 3D, this requires a very fine grid with several million of elements which is computationally highly expensive. When finer grid (about $400 \mathrm{~K}$ elements) is used, COMSOL runs for the entire simulation duration until the steady-state solution. The numerical oscillations can be significantly reduced but they do not completely disappear. The COMSOL results and the SA-steady solution are plotted in Figure 5. It can be clearly seen that the solutions are useless. Spurious Oscillations are spread over the whole domain. As mass transfer is more convective than heat ( $L e>1)$, oscillations are more pronounced for concentration contours than isotherms. The steady-state Nusselt and Sherwood numbers obtained using the semi-analytical solution and COMSOL (v5.3) are given in Table 2 which shows significant discrepancy between the results, especially for $\overline{S h}$. This is consistent with the results presented in Figure 5.

For the comparison between the SA-transient solution and COMSOL, we only investigate time variation of $\overline{N u}$ and $\overline{S h}$. For the sake of brevity, we do not present isotherms and concentration contours. The time variations of $\overline{N u}$ and $\overline{S h}$ are given in Figure 6. This figure indicates a huge discrepancy between the semi-analytical solution and COMSOL-v5.3. The latter produces unphysical results as both $\overline{N u}$ and $\overline{S h}$ are decreasing with time. The origin of this behavior is the spurious oscillations that appear during the entire simulation. 

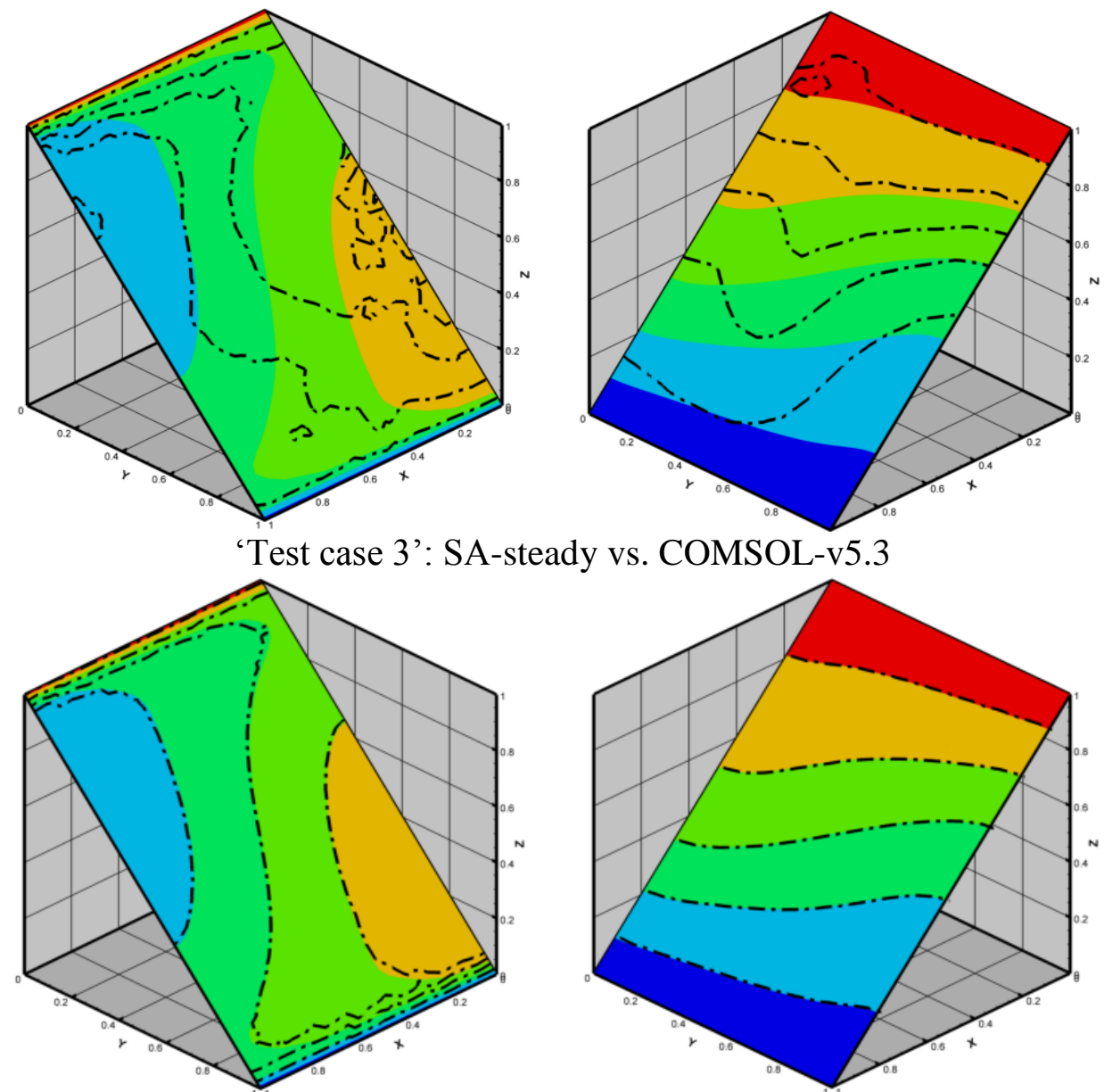

'Test case 3': SA-steady vs. COMSOL

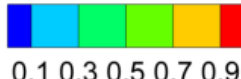

Figure 5. Comparison of the steady-state semi-analytical (SA-steady) solution in flood map and COMSOL in dashed lines for 'test case 3': concentration contours (left) and isotherms (right) in two diagonal plans. COMSOL-v5.3 is used to denote a previous version of the software while COMSOL is used for the newer release (v5.4). Parameters for 'test case 3' are given in Table 1.

We also simulate 'test case 3 ' using the new release of COMSOL (version 5.4) with a grid consisting of about $400 \mathrm{~K}$ elements. The resulting isotherms and concentration contours are plotted in Figure 5. This figure shows good agreement with the semi-analytical solution. In COMSOL (version 5.4) the software undergoes stability improvements that are introduced as updates. The results of Figure 5 confirm that the new numerical technique implemented in the new version of COMSOL allows for reducing the unphysical osciallations. But this technique cannot remove the osciallations competely, as some instabilities are still visible on the 
concentration contours (see Figure 5). Good agreement is also found between the SA-steady solution and COMSOL (version 5.4) reagarding $\overline{N u}$ and $\overline{S h}$, as given in Table 2 . The time variation of $\overline{N u}$ and $\overline{S h}$ with the new version of COMSOL are plotted in Figure 6. Excellent agreement can be observed with the SA-transient solution regarding $\overline{N u}$, while small discrepancy can be noted for $\overline{S h}$ which is underestimated in COMSOL. This means that the numerical scheme used in COMSOL overestimates the molecular diffusion. This phenomenon is known as numerical diffusion (Younes et al., 2007). It affects only $\overline{S h}$ because concentration distribution is sharper than the temperature distribution $(L e>1)$.

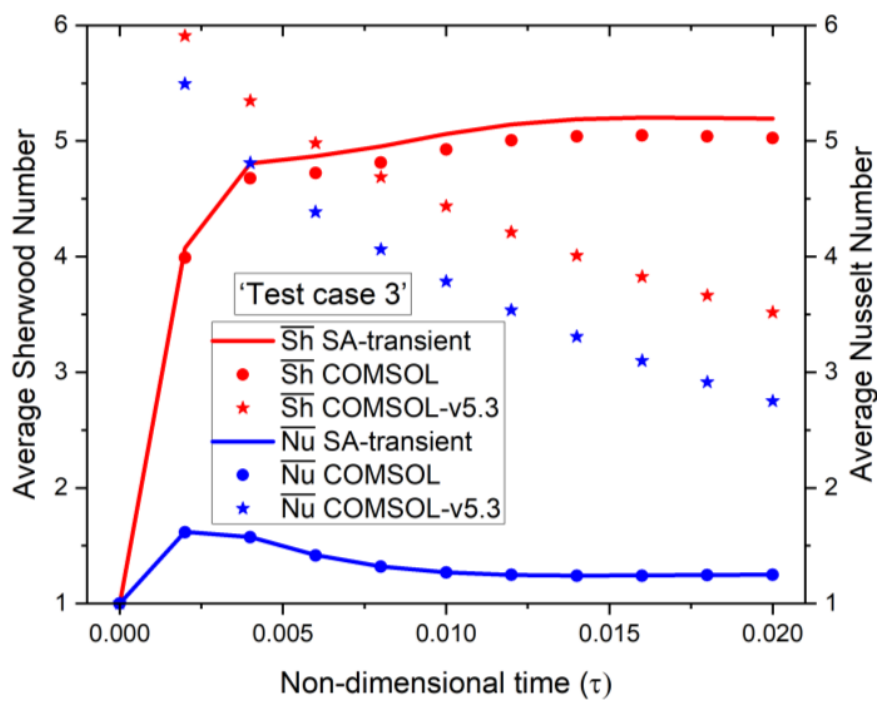

Figure 6. Time variation of the average Nusselt $(\overline{N u})$ and Sherwood $(\overline{S h})$ numbers: Comparison of the transient semi-analytical (SA-transient) solution and COMSOL for 'test case 3'. COMSOL-v5.3 is used to denote a previous version of the software while COMSOL is used for the newer release (v5.4). Parameters for 'test case 3' are given in Table 1.

Both versions of COMSOL (5.3) and (5.4) give similar results for 'test case 1' and 'test case 2'. Thus, contrary to the previous test cases, the numerical solution of 'test case 3 ' is sensitive to the numerical scheme used to solve the governing equations. This is an important property for a good benchmark that can be useful for the verification of numerical codes and to assess the 
robustness of numerical schemes for solving the equations of THC. 'test case 3' is also helpful in comparing performance of numerical codes. To highlight this property, we perform several simulations of 'test case 3' using different strategies of time integration in COMSOL. The results show that the performance of the numerical solution (CPU time) is more sensitive to the time integration scheme than the first test cases.

The comparison between the semi-analytical solution and COMSOL, in particular for 'test case 3', points out the high performance of the former. In fact, while a grid involving about $400 \mathrm{~K}$ elements (370K nodes), leading to a system with $740 \mathrm{~K}$ degrees of freedom, is required to obtain an accurate numerical solution, the semi-analytical solution is obtained with only $14 \mathrm{~K}$ degrees of freedom. This leads to a huge reduction in computational time. This gain is more significant for convection-dominated cases. Moreover, we should mention that the problem of unphysical oscillations has been reported in purely solute or thermal natural convection in porous media at relatively high Rayleigh number $(\mathrm{Ra}=500)$ (Shao et al., 2018). The results here show that THC simulation is more challenging than purely compositional or thermal natural convection because numerical instabilities could appear at relatively small values of Rayleigh number.

\section{Sensitivity to parameters for THC under crossed thermal and solute gradients}

At steady-state, the THC in the porous box is controlled by three parameters: $R a_{T}, N_{g}$ and $L e$. Two additional parameters ( $\varepsilon$ and $\sigma$ ) are involved in transient solutions. The developed semianalytical solution deals with three-dimensional THC in the case of crossed-horizontal salinity and temperature gradients. The effects of the parameters controlling THC on heat and mass transfer processes in such a case have never been investigated in the literature because it requires 
3D simulations which are computationally expensive. In this section, taking advantage of the efficiency and robustness of the developed semi-analytical solution, we perform a detailed parameters sensitivity analysis. The effects of $\varepsilon$ and $\sigma$ on convective flow and heat and mass transfer are predictable as they mainly affect the time required to reach the steady-state regime. Thus, in our analysis we consider the sensitivity to $R a_{T}, N_{g}$ and $L e$. For the transient simulations, we assume constant $\varepsilon=0.1$ and $\sigma=0.46$.

\subsection{Effect of $R a_{T}$}

We assume moderate value of $L e(=2)$ to confine the discussion. This value is physically plausible as heat transfer is more diffusive than mass transfer. We vary $R a_{T}$ from 10 to 200 and we consider two cases dealing with thermal $\left(N_{g}=0.5\right)$ and solute $\left(N_{g}=1.5\right)$-dominated convective flow, respectively. The cases dealing with thermal-dominated convective flow are denoted by 'TD' while the cases involving solute-dominated convective flow are denoted by 'SD'. The effect of $R a_{T}$ on the structure of the steady-state velocity field is investigated using the arrow-surface representation as in Figure 7. This figure is helpful in understanding the 3D structure of the main flow which is the superposition of the two components. The first component is related to the solute-driven convective flow (SDC) while the second one is the thermally driven convective flow (TDC). The SDC flow component is two-dimensional and occurs in the vertical planes orthogonal to the x-axis. The TDC flow component is also planar and takes place in the vertical planes orthogonal to $\mathrm{y}$-axis (see figure $\mathrm{C} 1$ in Appendix $\mathrm{C}$ ). The superposition of these flow components (SDC and TDC) results in a three-dimensional overall flow. Different structures are reported for the TD and SD cases. For the TD case, the primary flow is the TDC flow component, which is two-dimensional. The three-dimensional structure of 
the overall flow is created by the crossing SDC flow component that leads to vortex formation at the vertical plane $Y=1$. The inviscid evolution of this vortex within the domain is represented using stream-tubes in Figure 7 (bottom figures). For the TD case, the vortex structure is an association of two hourglass shaped vortices oriented diagonally from the plane $Y=1$ to the plane $\mathrm{Y}=0$. For the SD case, the primary flow is analogue to the SDC flow component and threedimensional structure is attributed to the TDC flow component. Crossing-flows lead to a central vortex which has the same shape as in the SD case, but with a different orientation from the plane $X=1$ to $X=0$. The iso-surfaces of the steady-state vector potential are given in Appendix $C$ (Figure C2). This figure indicates that distribution of $\Psi_{X}$ is attributed to the SDC flow. This is consistent with equation (17) which confirms that variation of $\Psi_{X}$ is mainly related to the ycomponent of the concentration gradient. The variation of $\Psi_{Y}$ is linked with the TDC which is consistent with the mathematical formulation in equation (18). For low a Rayleigh number, the vector potential isosurfaces have a regular form, indicating almost two-dimensional conditions. Irregular shapes can be observed at high Rayleigh number, indicating three-dimensional flow. For the TD case, $\Delta \Psi_{X}(=0.09)$ is smaller than $\Delta \Psi_{Y}(=0.18)$. Thus, the TDC flow is dominating. The opposite is true for the SD case. Vector potential isosurfaces indicate that the strength of the convective flow increases with increasing Rayleigh number (both $\Delta \Psi_{X}$ and $\Delta \Psi_{Y}$ increase with $R a_{T}$ ). 

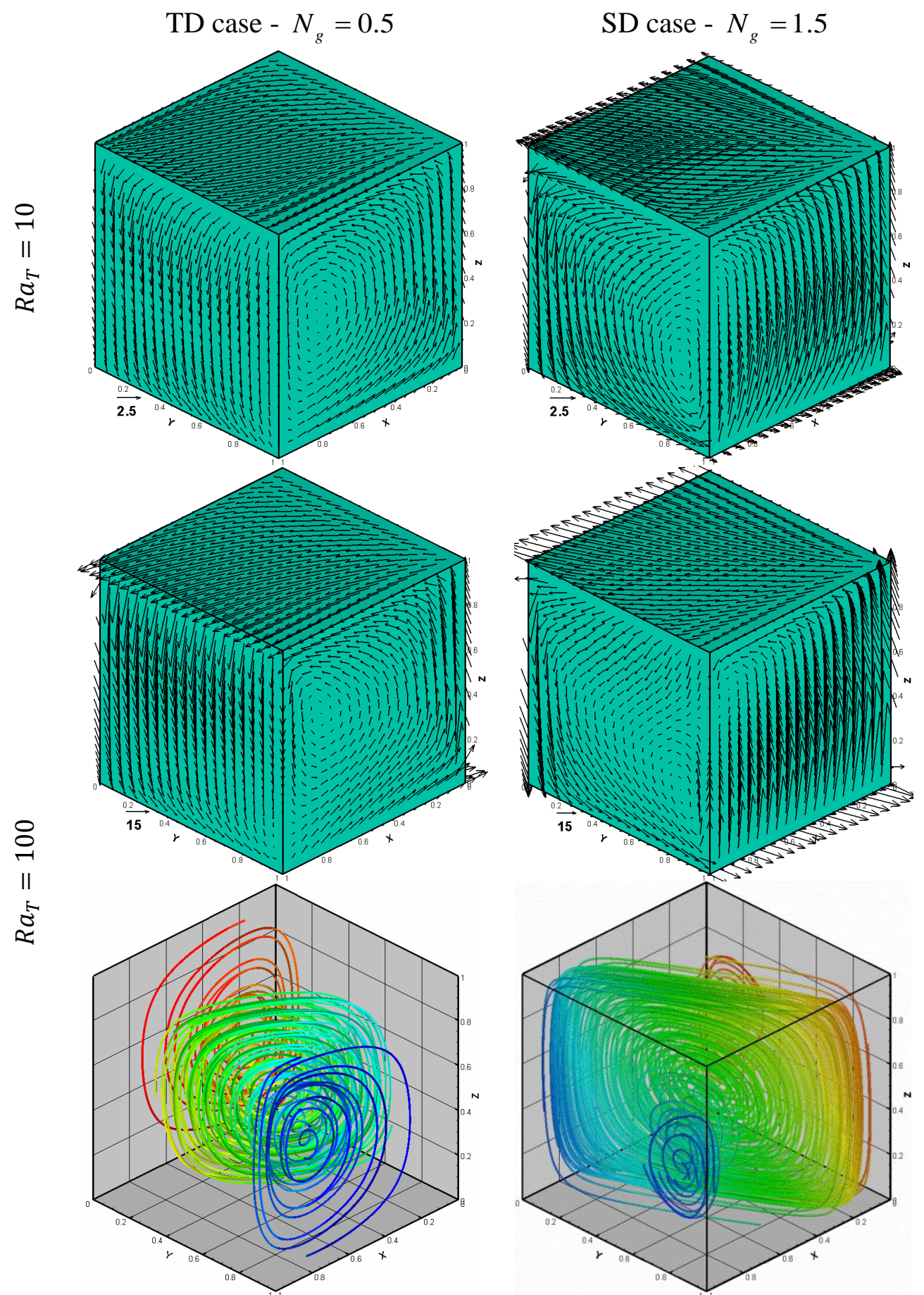

Figure 7. Effect of Rayleigh number on the flow structure: Arrow surface plot of the steady-state velocity field at small and large thermal Rayleigh number in the cases of thermal (TD) and solute (SD) -dominated convective flow. Bottom figures: Stream-tubes showing the behavior of the vortex within the domain. For the TD case the stream-tubes are highlighted with concentration while for the SD cases they are highlighted with temperature. 
Figure 8 shows the effect of $R a_{T}$ on the concentration and temperature distributions. These figures indicate that concentration distribution is sensitive to $R a_{T}$ in the $\mathrm{SD}$ case while temperature field is affected by $R a_{T}$ in the TD case. At low $R a_{T}$, in the TD case, the concentration isosurfaces are almost planar and vertical because the main flow occurs in the planes orthogonal to the concentration gradient. This is also the case for temperature isosurfaces at low $R a_{T}$ in the SD case. At high $R a_{T}$, in the TD case, both temperature and concentration isosurfaces follow the flow structure. The three-dimensional variation of the concentration is confined to limited zones at the top and bottom surfaces of the domain. However, the temperature isosurfaces are fully three-dimensional. The opposite is true for the SD case at high thermal Rayleigh number.

$$
\text { TD case }-N_{g}=0.5
$$

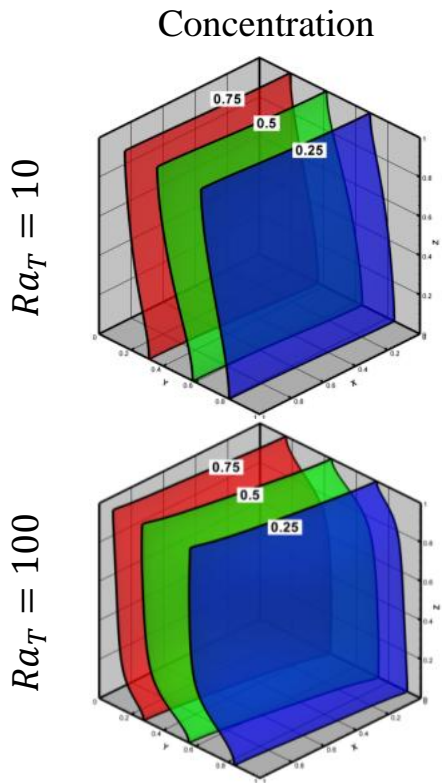

Temperature

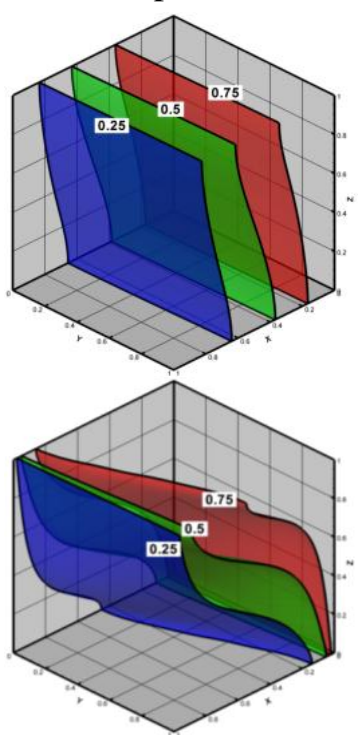

SD case $-N_{g}=1.5$

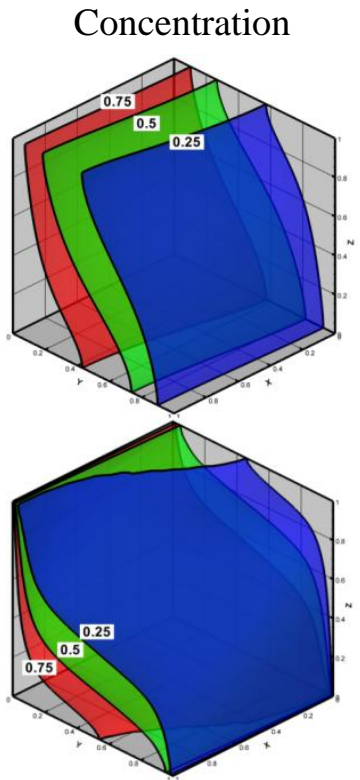

Figure 8. Effect of the Rayleigh number on the concentration and temperature distributions: Steady-state concentration and temperature isosurfaces $(0.25,0.5$ and 0.75$)$ at small and large thermal Rayleigh numbers in the cases of thermal (TD) and solute (SD) -dominated convective flow. 
The effects of $R a_{T}$ on $\overline{N u}$ and $\overline{S h}$ (both steady-state and transient) are depicted in Figure 9, for both TD and SD cases. Figures 9a and 9b confirm that the steady-state values of both $\overline{N u}$ and $\overline{S h}$ increase with $R a_{T}$. The increase of Rayleigh number leads to flow intensification which narrow the solute and thermal boundary layers and enhance heat and mass transfer to the domain. However, the effect of $R a_{T}$ on heat $(\overline{N u})$ and mass $(\overline{S h})$ transfer fluxes are not equally distributed. In the TD case, $R a_{T}$ has a slight effect on $\overline{S h}$ and a significant impact on $\overline{N u}$. The opposite is true for the SD case. This means that in the TD cases, the increase of $R a_{T}$ significantly enhance the heat transfer flux, while for the SD cases, $R a_{T}$ has more impact on the mass transfer flux. 


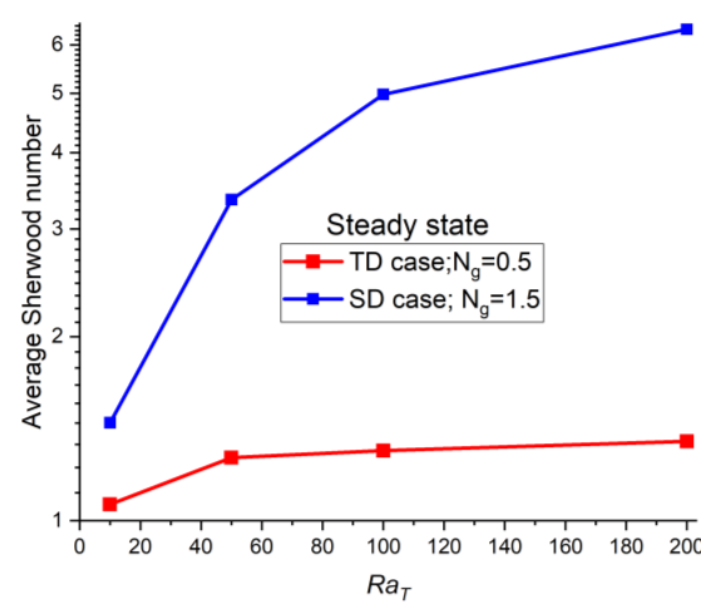

(a)

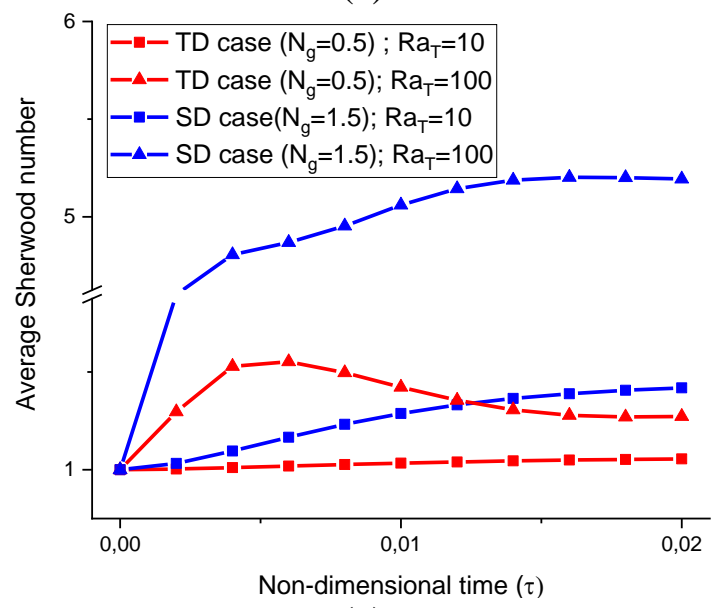

(c)

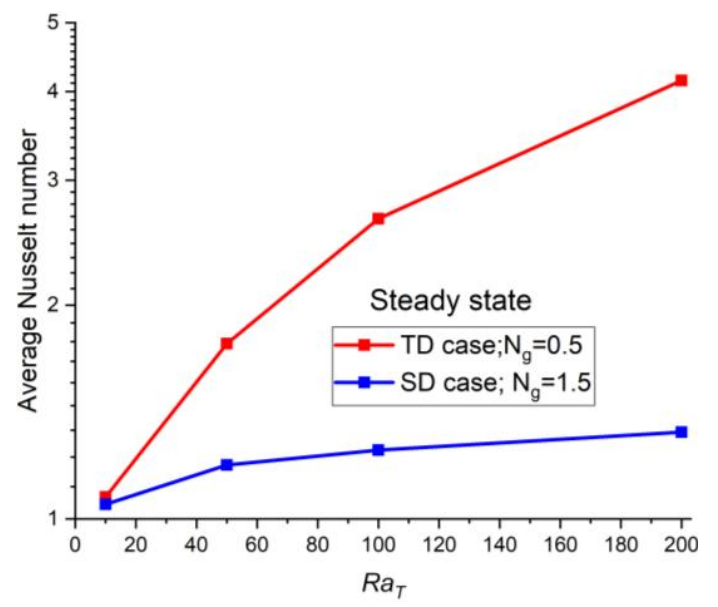

(b)

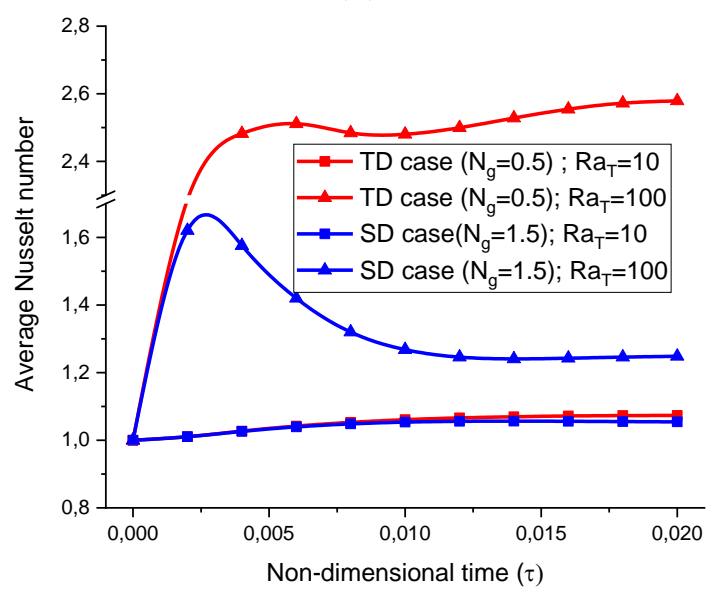

(d)

Figure 9. Effects of the thermal Rayleigh number on the average Sherwood and Nusselt numbers in the cases of thermal (TD) and solute (SD) -dominated convective flow: Steady-state regime (a and $b$ ) and transient regime (c and d).

Figures $9 \mathrm{c}$ and $9 \mathrm{~d}$ show the time variations of $\overline{S h}$ and $\overline{N u}$ at low and high values of $R a_{T}$, for

TD and SD cases. The general behavior is that both $\overline{S h}$ and $\overline{N u}$ start from 1 at $\mathrm{t}=0$ and evolve until reaching asymptotic values indicating the steady-state regime. The initial value of one is related to the initial conditions that are linearly distributed for both concentration and temperature. The time required to reach the steady-state regime decreases with increasing $R a_{T}$. While in general, the mass flux to the cavity increases with time, Figure 9c shows particular variation of $\overline{S h}$ in the TD case and at high Rayleigh number. This figure shows that, in such a 
case, the time variation of the mass flux (as measured by $\overline{S h}$ ) exhibits two regimes. Thus, it increases first to reach a maximum value at a transition time after which it decreases asymptotically toward the steady-state value. A similar behavior can be observed for $\overline{N u}$ but in the SD case and at high Rayleigh numbers.

\subsection{Effect of $N_{g}$}

As in the previous section, we assume $L e=2$. Since $N_{g}$ affects mainly the buoyancy forces, we consider high convective cases by assuming $R a_{T}=100$. The effect of $N_{g}$ on the flow structure was investigated in the previous section. Thus, we investigate its effect on the maximum velocity components $\left(Q_{X}^{\max }, Q_{Y}^{\max }\right.$ and $\left.Q_{Z}^{\max }\right) \cdot \boldsymbol{Q}=\left(\mathbf{q} \times \frac{\alpha}{H^{2}}\right)$ is the non-dimensional velocity field. The steady-state results (Figure 10a) show that the maximum velocity component is in the vertical direction $\left(Q_{Z}^{\max }\right)$. There is an enhancement in the vertical flow when $N_{g}$ is increased. In the TD cases, the $x$-component decreases with $N_{g}$ while the $y$-component increases. Both components increase with $N_{g}$ in the SD cases. For the TD cases, the flow in the $x$-direction is higher than in the $y$-direction. The opposite is true in the SD cases. The average Nusselt number decreases with $N_{g}$ while the average Sherwood number increases (Figure 10b). This indicates that the increase of $N_{g}$ leads to the improvement of the mass flux to the domain and to the diminishment of the heat flux.

The transient solutions confirm that the increase of $N_{g}$ leads to the enhancement of the flow in the $y$-and $z$-components, whatever the time (Figure 10c). It is also true for the $\mathrm{x}$-component in the SD cases and TD cases, but in TD cases, just for a short time at the beginning of the transient regime. After this short time and until the steady-state regime, $Q_{X}^{\max }$ decreases with the increase 
in $N_{g}$. In general, these results are consistent with the flow behavior in the steady-state regime. Figure 10c shows that $Q_{z}^{\max }$ increases with time and reaches the steady-state regime faster than the other velocity components. $Q_{Y}^{\max }$ is almost increasing with time, but less time variability can be observed in the TD cases. Variation of $Q_{X}^{\max }$ in time is highly sensitive to $N_{g}$. We can observe in Figure 10c that, in the TD case, $Q_{x}^{\max }$ increases with time for $N_{g}=0.25$ while it decreases when $N_{g}$ is increased to 0.75 . For the SD cases with $N_{g}=1.25, Q_{X}^{\max }$ exhibits two regimes for time variation as it increases for a short time at the beginning of the simulation and then it decreases to reach the steady-state value. For the SD cases but with $N_{g}=1.75, Q_{x}^{\max }$ decreases with time. The time variations of the average Sherwood and Nusselt numbers are given in Figures $10 \mathrm{~d}$ and $10 \mathrm{e}$, respectively. It is clear that, whatever the time, $\overline{S h}$ increases with the increase of $N_{g}$, while $\overline{N u}$ is decreasing. In the TD cases, $\overline{S h}$ follows two regimes of time variation. It increases with time at the beginning of the transient regime and then decreases until reaching its steady-state value. Similar behavior is observed for $\overline{N u}$ (Figure 10e), except for the TD case at small Gravity number $\left(N_{g}=0.25\right)$. 


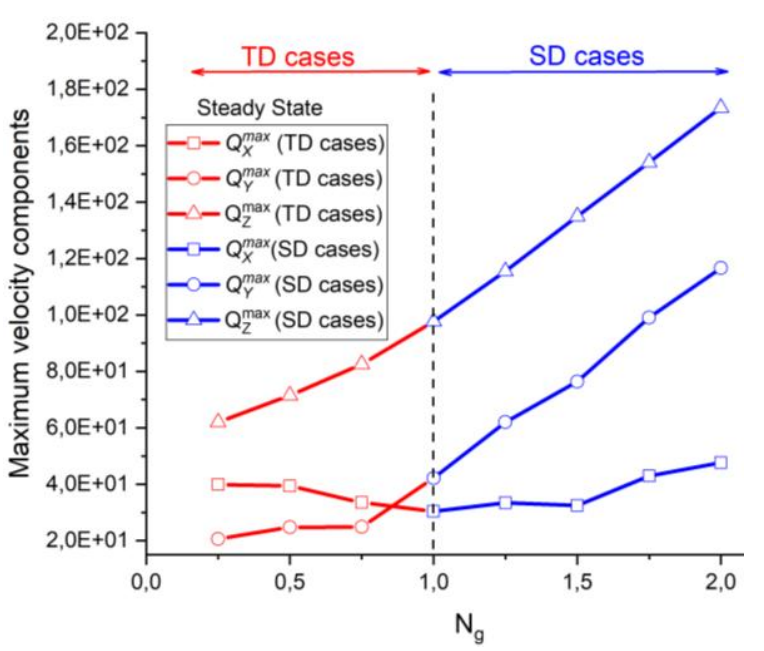

(a)

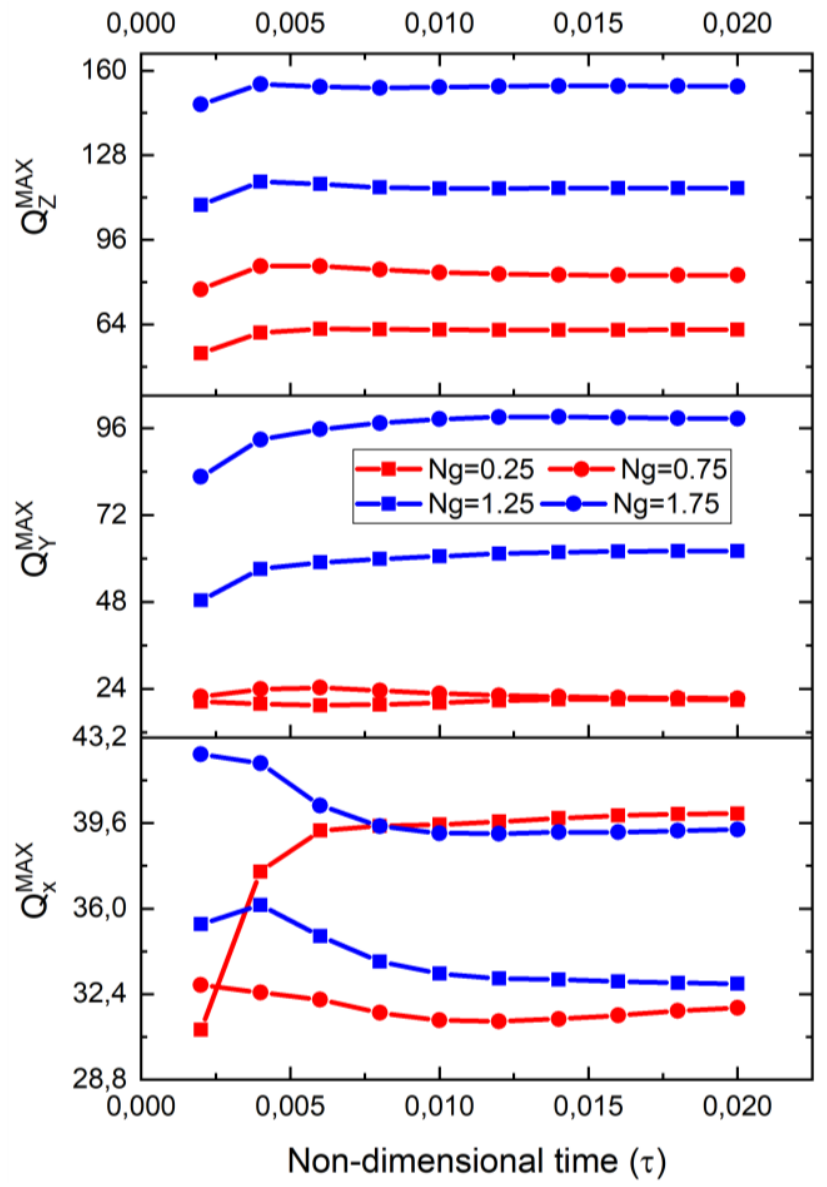

(c)

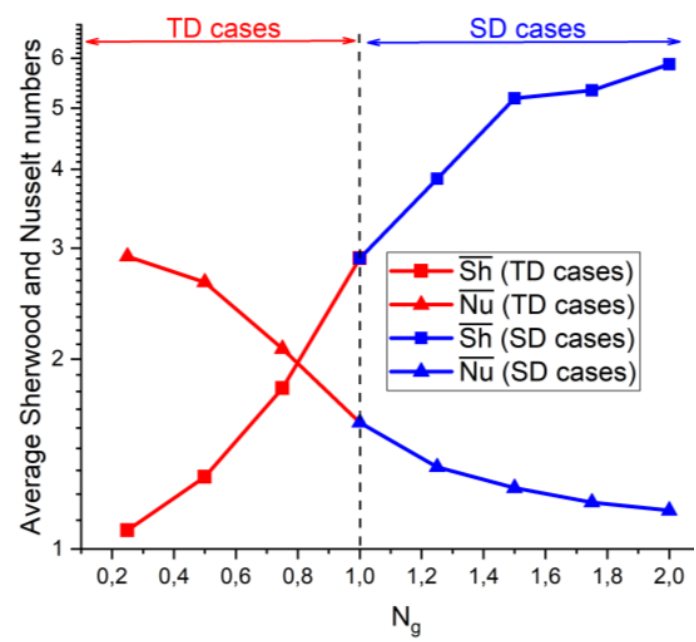

(b)

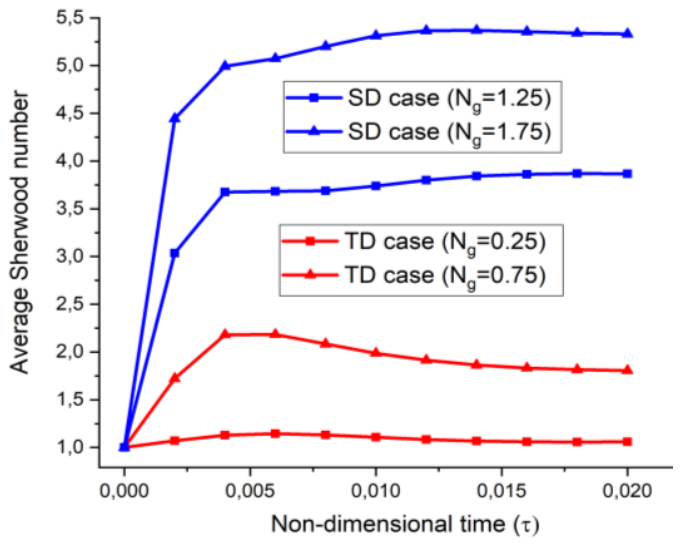

(d)

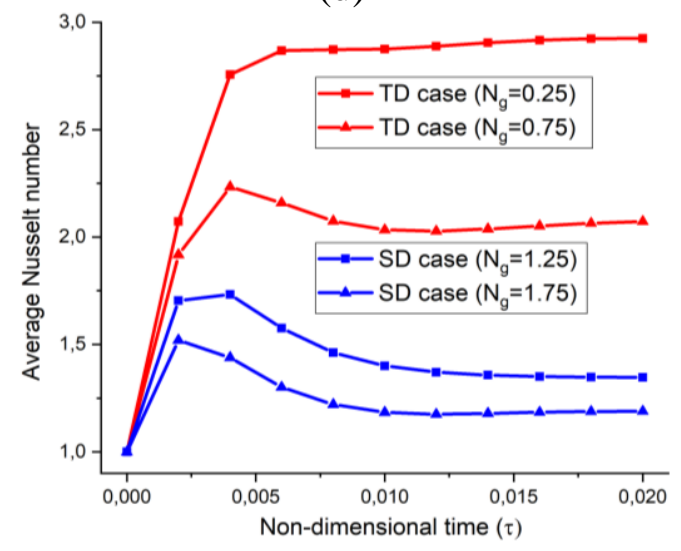

(e)

Figure 10. Effects of the Gravity number on the maximum velocity components, average Sherwood and Nusselt numbers for steady-state ( $a$ and b) and transient (c-e) regimes.

The effects of $N_{g}$ on concentration and temperature distributions are shown in Figure 11. For the TD cases, at constant $R a_{T}$, the increase in $N_{g}$ can be interpreted as an increase in the 
concentration gradient. This enhances the convective flow in the vertical planes parallel to YOZ and reduces the flow component in the vertical planes parallel to $\mathrm{XOZ}$ (related to the thermal gradient). This explains why the concentration isosurfaces become deformed when $N_{g}$ is increased while the opposite is true for the temperature isosurfaces. Similar behavior is observed for the SD cases.
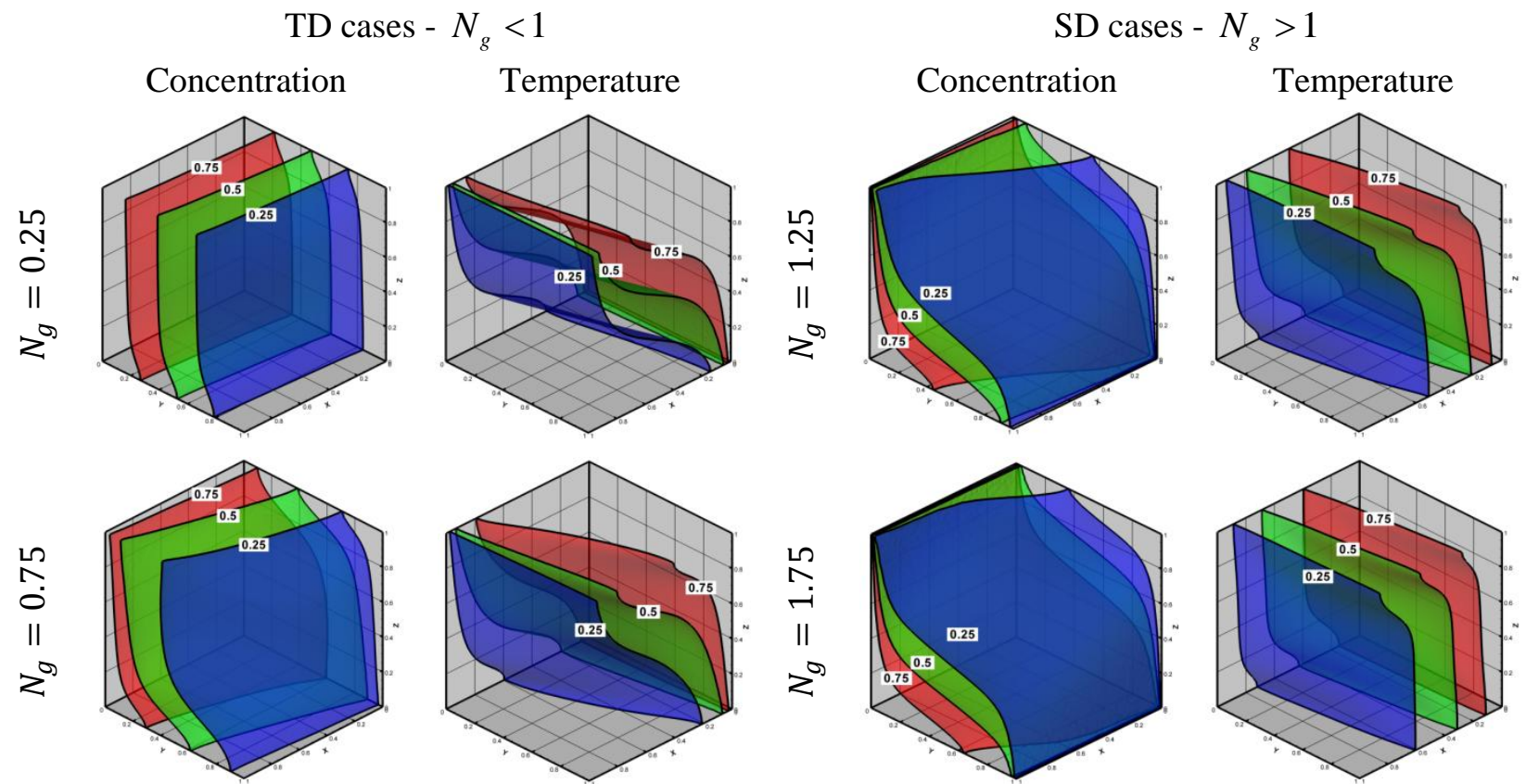

Figure 11. Effect of the Gravity number $\left(N_{g}\right)$ on the concentration and temperature distributions: Main temperature and concentration isosurfaces $(0.25,0.5$ and 0.75$)$ for different values of $N_{g}$ in the cases of thermal (TD- left) and solute (SD- right) -dominated convective flow. 


\subsection{Effect of $L e$}

The effect of $L e$ is investigated in both TD $\left(N_{g}=0.5\right)$ and SD $\left(N_{g}=1.5\right)$ cases. We assume $R a_{T}=100$. The effects of $L e$ on the steady-state maximum velocity components are given in Figure 12a. It can be seen that the velocity field is slightly sensitive to $L e$ in the TD cases. At constant thermal Rayleigh number, the increase in $L e$ can be interpreted as a decrease of the molecular diffusion coefficient. The latter can lead to a sharper concentration distribution and, as a result, a higher solute gradient. However, since convection flow is mainly caused by the thermal gradient, the increase of the solute gradient does not affect the velocity field. For the SD case, where convective flow is mainly related to concentration gradient, the velocity field becomes sensitive to $L e$, in particular $Q_{Y}^{\max }$ and $Q_{Z}^{\max } . Q_{X}^{\max }$ remains slightly sensitive to $L e$, which is logical as this component of the flow is caused by the temperature gradient. Figure 12a shows an intensified convection rotating flow with the increase in Le. Time variations of the maximum velocity components are given in Figure 12c. This figure confirms that, in the TD cases, the transient convective flow is slightly sensitive to $L e . Q_{X}^{\max }$ increases with time to reach its steady-state value while $Q_{Z}^{\max }$ and $Q_{Y}^{\max }$ have critical time for the transition between increasing and decreasing variations. For the SD case, as for the steady-state regime, the transient maximum velocity components increase with the increase of $L e$. It can be seen that $Q_{Y}^{\max }$ and $Q_{Z}^{\max }$ increase with time until reaching the steady-state values, while $Q_{X}^{\max }$ has different behavior as it is decreasing. In the SD case, both mass and heat fluxes to the domain are sensitive to $L e$ (Figure 12b). As expected the mass flux to the domain is enhanced with the increase in $L e$ . This is attributed to the reduction in the solute gradient. However, the heat flux to the domain is reduced. In the TD case, $\overline{N u}$ is slightly sensitive to $L e$ while expected $\overline{S h}$ increases with the 
increase of $L e$. The time variations of $\overline{S h}$ and $\overline{N u}$ are given in Figures $12 \mathrm{~d}$ and 12e, respectively. Similar to the steady-state condition, the transient mass flux in the domain increases with the increase in $L e$ while the transient heat flux decreases. In the SD case, $\overline{S h}$ increases with the time until reaching the steady-state regime while in the TD case it evolves to the steady-state value by following increasing and decreasing periods. The opposite is true for $\overline{N u}$. It is relevant to mention that, while steady-state heat flux has been found to be insensitive to $L e$, the transient behavior of the $\overline{\mathrm{Nu}}$ shows some sensitivity to this parameter. Figures $12 \mathrm{~d}$ and $12 \mathrm{e}$ show that both $\overline{S h}$ and $\overline{N u}$ reach the steady-state regime in the SD case faster than in the TD case.

The effects of $L e$ on the temperature and concentration isosurfaces are shown in Figure 13. It is clear from this figure that, for the considered range of variations, in both SD and TD cases, the temperature and concentration fields are slightly sensitive to Le. More sensitivity is observed for concentration than temperature. 


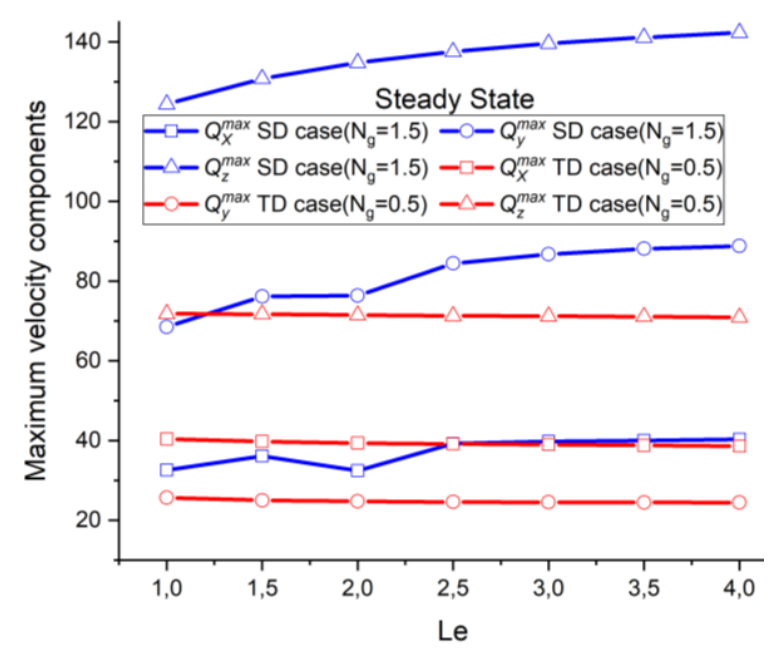

(a)

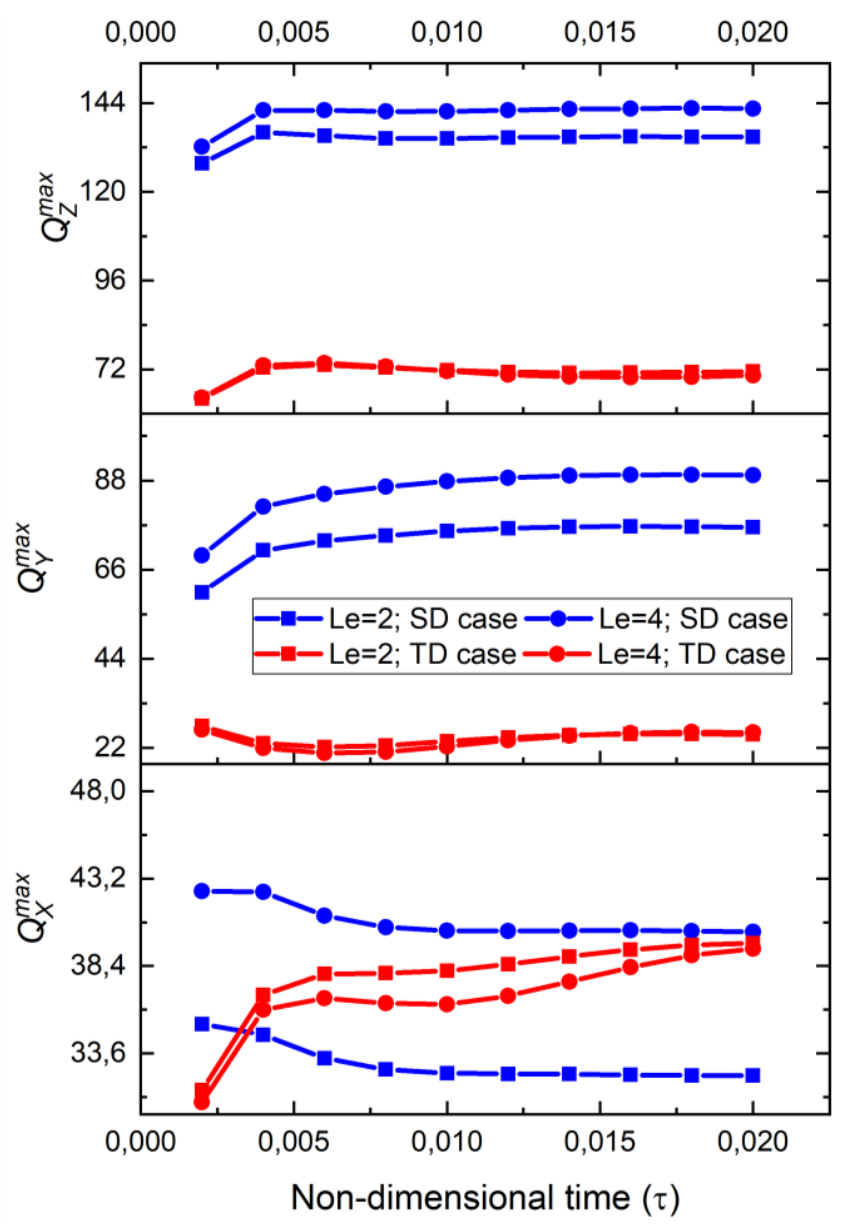

(c)

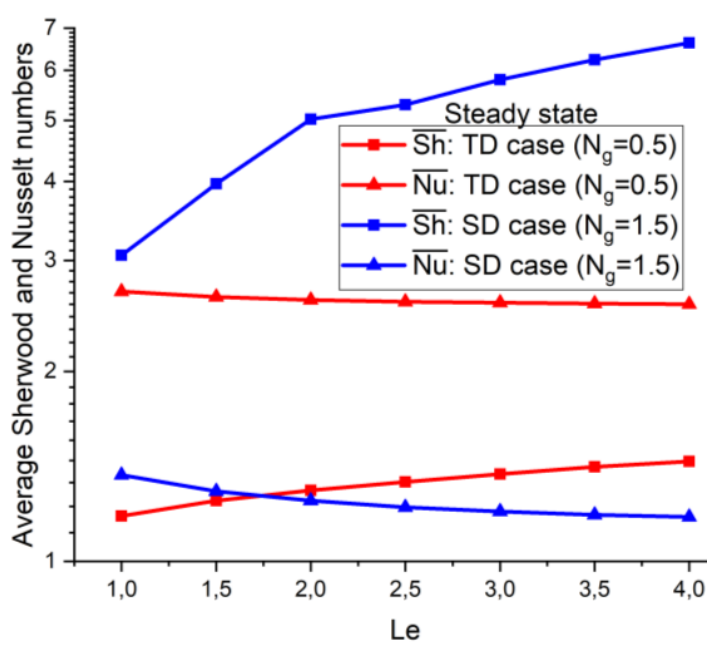

(b)

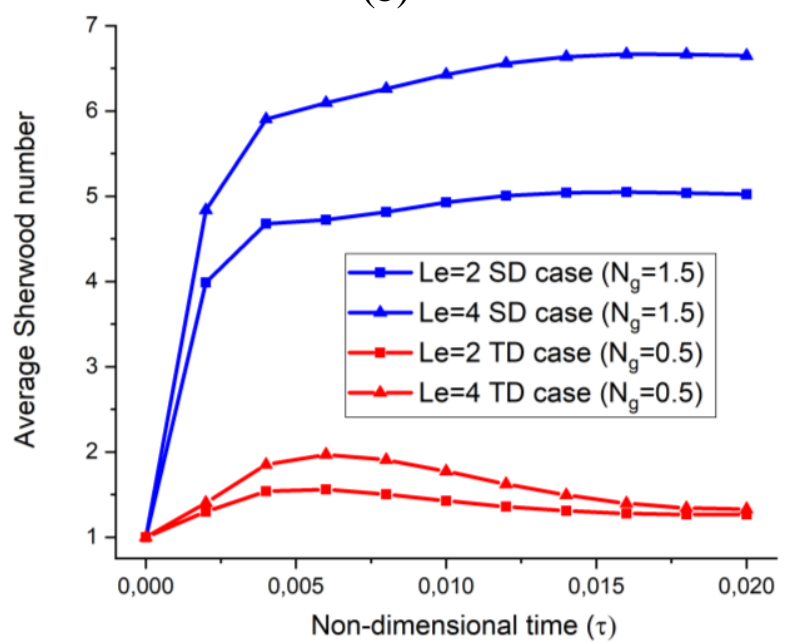

(d)

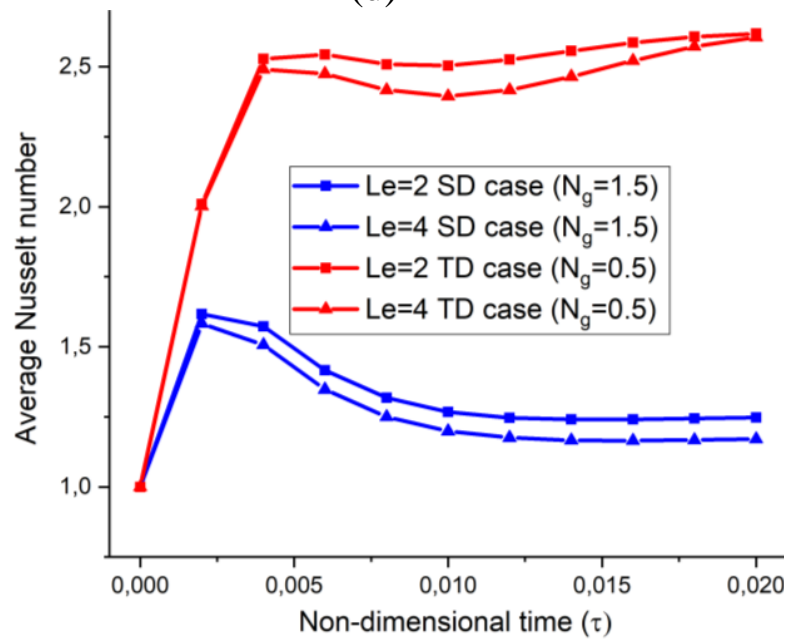

(e)

Figure 12. Effects of Lewis number on the maximum velocity components, average Sherwood and Nusselt numbers for steady-state ( $\mathrm{a}$ and $\mathrm{b}$ ) and transient (c-e) regimes. 

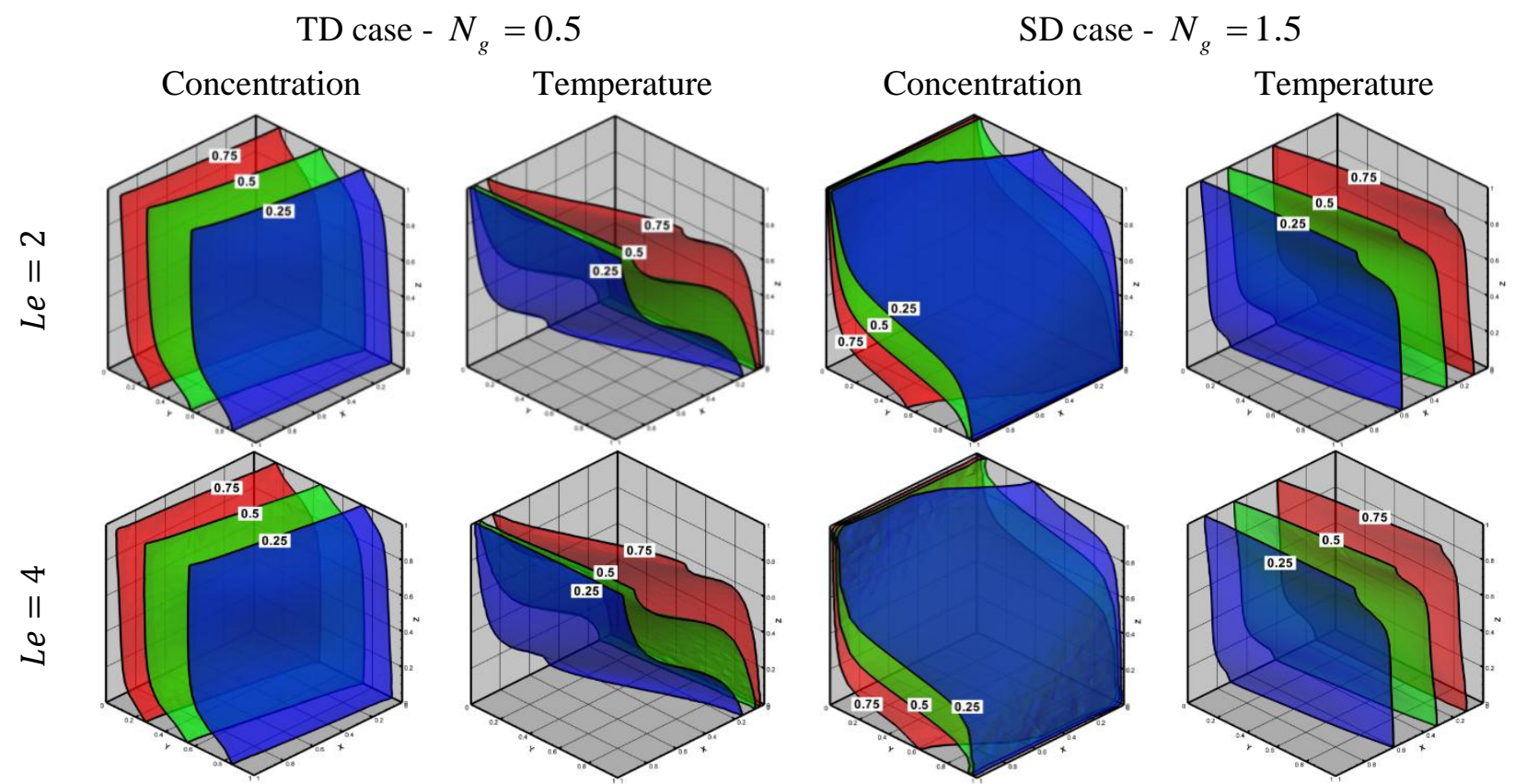

Figure 13. Effect of the Lewis number on the concentration and temperature distributions: Steady-state concentration and temperature isosurfaces $(0.25,0.5$ and 0.75$)$ at different values of Lewis numbers in the TD and SD cases.

\section{Understanding the effects of temperature and concentration gradients on heat and salinity fluxes}

Several applications involve THC processes under different configurations of heat and salinity gradients. For instance, in applications involving injection of hot fluids inside wells (i.e geothermal, gas and oil wells) and in steam injection, there are significant heat losses that create a temperature gradient in the aquifers. In salinized aquifers, this creates an interaction with the existing salinity gradient (van Lopik et al., 2015). Thus, in such a case, it is important to understand the effect of temperature gradient on mass flux entering the domain and the impact of salinity gradient on heat flux, which is the main objective of this section. This is useful to investigate the heat losses caused by the salinity gradient and/or the salinization induced by the heat gradient. Several previous studies addressed this question but under a 2D assumption (van Lopik et al., 2015), which is not valid in the case of crossed-horizontal temperature and salinity gradients because of the 3D effects. We address this question taking advantage of the developed 
3D semi-analytical solution. Thus, we first assume variable-temperature gradient $\Delta T=T_{H}-T_{C}$ and we investigate the effect of $\Delta T$ on mass flux, as measured by the average Sherwood number. All other parameters related to fluid and porous domain properties are assumed to be constant. The analysis is based on the parameters used in 'test case 3', as described in Table 1. However, to investigate both TD $\left(N_{g}<1\right)$ and SD $\left(N_{g}>1\right)$ cases, we consider $N_{g}=5$ for $\Delta T=1$, we then increase $\Delta T$ from 1 to 11 , progressively. This corresponds to the decrease in $N_{g}$ from 5 to 0.45 . The variation of the steady-state average Sherwood number with respect to $\Delta T$ is depicted in Figure 14a. As it can be seen, the increase of $\Delta T$ reduces the salinity flux in both TD and SD cases. In fact, the increase in $\Delta T$ is associated with the enhancement of the convective flow caused by the thermal gradient which occurs in the vertical planes parallel to the salinized wall. This leads to a reduction in the flow component perpendicular to the salinized wall and, as a result, reduces the salinity flux. Figure 14a shows that the salinity flux is more sensitive to the temperature gradient in the SD cases than the TD cases. The effect of $\Delta T$ on the transient behavior of the salinity flux is depicted in Figure 14b. For small temperature gradient, the salinity flux reaches the steady-state value faster than high temperature gradient where twotime variation regimes (increasing then decreasing) can be observed. As for steady-state, the transient behavior of the salinity flux is slightly sensitive to $\Delta T$ in the TD cases. We also investigate the effect of concentration gradient $\left(\Delta c=c_{s}-c_{f}\right)$ on heat flux, as measured by the average Nusselt number. Thus, as for the effect of $\Delta T$, we keep all parameters constant and we increase $\Delta c$ from 1 to 11 . We use the same parameters as in 'test case 3' in Table 1, but we assume that $N_{g}=0.2$ for $\Delta c=1$. Thus $N_{g}$ varies from 0.2 to 2.2 . Figure $14 \mathrm{c}$ shows that the steady-state heat flux to the domain decreases with increasing $\Delta c$. The explanation of this 
behavior is analogous to the variation of the salinity flux with temperature gradient. The heat flux is more sensitive to $\Delta c$ in the TD cases. Figure $14 \mathrm{~d}$ indicates that, in the SD cases, the transient heat flux reaches the steady-state regime faster than the TD cases. In contrast to the salinity flux, similar behavior of heat flux with respect to time can be observed, whatever the concentration gradient.

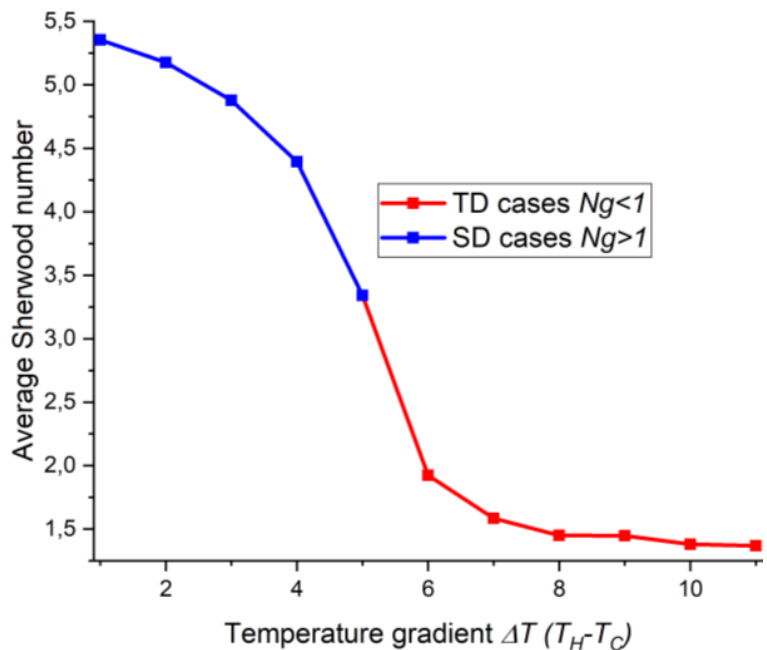

(a)

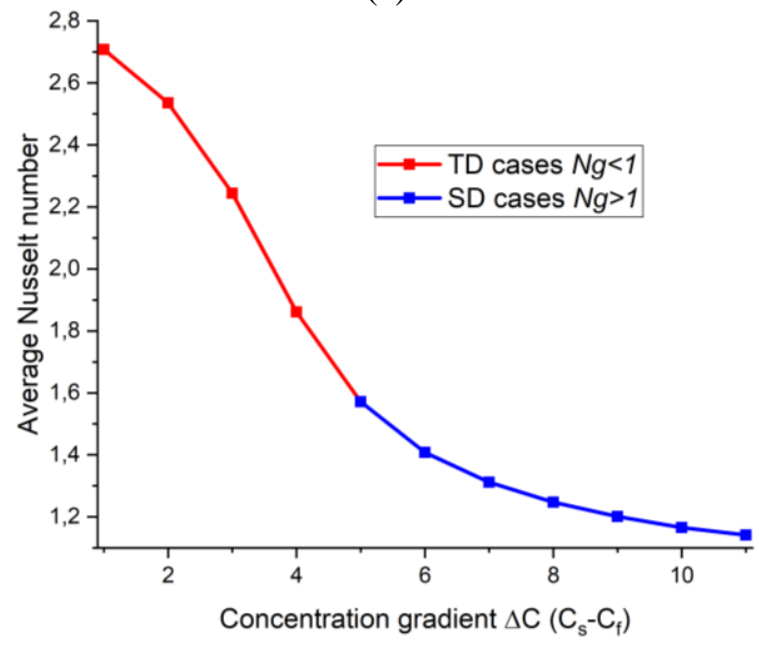

(c)

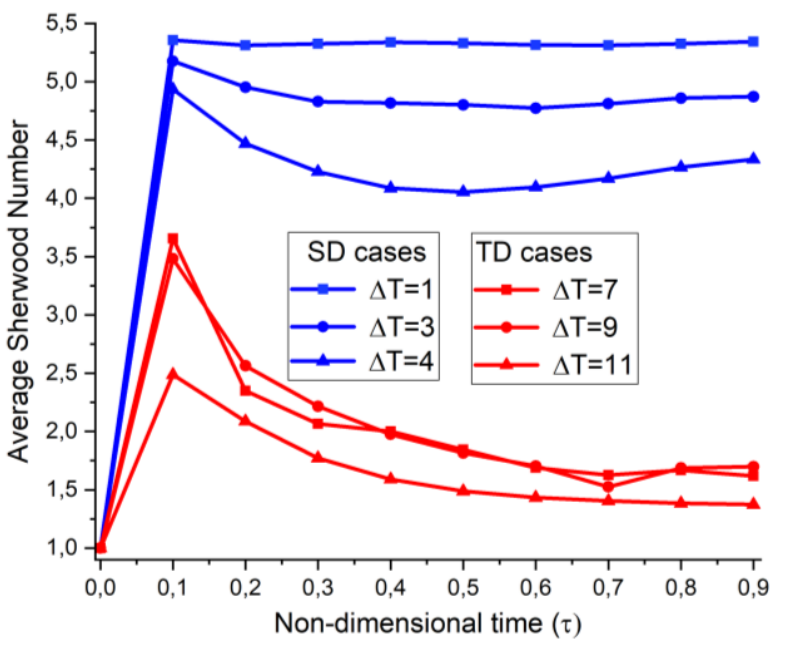

(b)

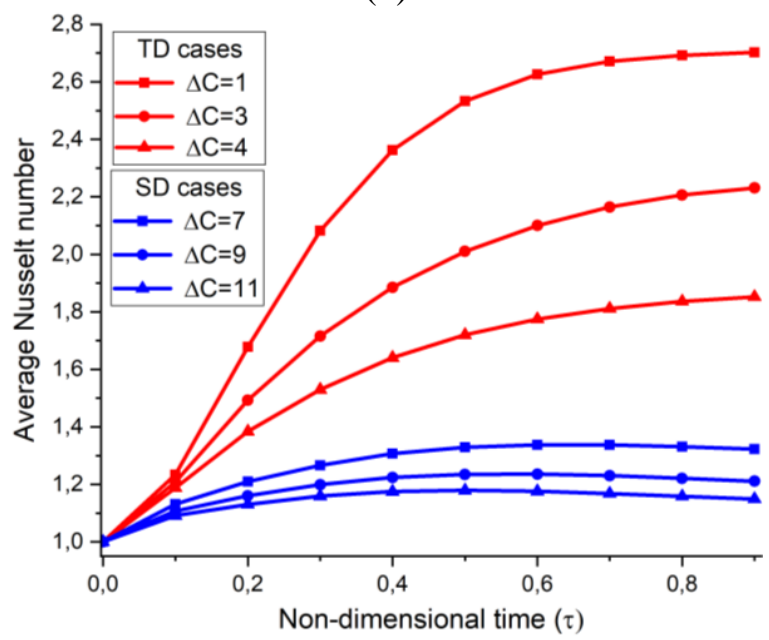

(d)

Figure 14. Effect of temperature (resp. concentration) gradient on mass (resp. heat) flux to the domain, as measured by the average Sherwood (resp. Nusselt) number: Steady-state regime (a and c) and transient regime (b and d). 


\section{Conclusion}

In this work, we investigated three-dimensional THC in a porous box under both transient and steady-state regimes. We consider the case of crossed-horizontal temperature and concentration gradients for which the $2 \mathrm{D}$ assumption is not valid. We develop a meshless semi-analytical solution based on the Fourier series method (FS). From a theoretical and technical point of view, the main contribution and novelty of this work is the new implementation of the FS method for solving transient problems. The governing flow, mass and heat transport equations have been amenable to a FS solution by using the vector potential, the $3 \mathrm{D}$ counterpart of the stream function. The FS method leads to a spectral system of differential-algebraic equations. In the spectral space, we express the flow (i.e. vector potential) in terms of the concentration and temperature Fourier series and we convert the spectral system to a system of ordinary differential equations. This allows for reducing the number of degree of freedom and improves the resolution procedure as it is well-known that ordinary differential systems are more stable than differentialalgebraic systems.

The semi-analytical solution is verified against a finite element solution obtained with COMSOL. In general the semi-analytical solution provides high accurate results with reduced number of degree of freedom. Excellent agreement between these solutions has been observed for cases involving small Rayleigh number. Numerical experiments with a high Rayleigh number show high sensitivity of the finite element solution to the computational mesh and the numerical technique used in space discretization and time integration. This highlights the worthiness of the developed semi-analytical solution as a benchmark for the assessment of newly developed numerical methods and schemes for THC problems. We provide high quality data, based on quantitative metrics, which can be used for benchmarking numerical codes. 
We performed a complete parameter sensitivity analysis to investigate the effect of governing parameters on the THC processes. In our analysis we distinguish between the cases of thermaldominated convective flow (TD) and solute-dominated convective flow (SD). In both cases we observed the formation of single vortex convective flow which cannot be captured in 2D cases. The orientation of the vortex flow depends on the Gravity number $\left(N_{g}\right)$ while its intensity is also sensitive to the thermal Rayleigh number $\left(R a_{T}\right)$. As it is well known in problems involving density driven flow, the increase of $R a_{T}$ intensifies the convective flow and leads to the increase of both steady-state Nusselt $(\overline{N u})$ and Sherwood $(\overline{S h})$ numbers. In the TD cases, two regimes (increasing then decreasing) of transient behavior are observed for $\overline{S h}$. Similar transient evolution is observed for $\overline{N u}$ in the SD cases. The increase of the Gravity number can be interpreted as an increase of the solute Rayleigh number which is accompanied by convective flow intensification. The flow acceleration occurs in the plane parallel to the concentration gradient. This leads to the enhancement of the steady-state salinity flux entering the domain (i.e. $\overline{S h}$ ) and reduction of the heat flux (i.e. $\overline{N u}$ ). Transient evolutions of $\overline{N u}$ and $\overline{S h}$ are sensitive to $N_{g}$. Either monotonic increasing variation or two regimes of variation can be observed, depending on $N_{g}$. The convective flow is slightly sensitive to the Lewis number $(L e)$. But in general, the vertical component of flow increases with Le. The steady-state heat flux to the domain is also slightly sensitive to $L e$, especially in the TD cases, while the transient evolution of the heat flux is highly sensitive to this parameter.

We also investigate the effect of thermal gradient on the salinity flux and the effect of concentration gradient on heat flux. The results show that, in the case of crossed gradients, the 
increase in heat gradient reduces the salinity flux to the domain, especially in the SD cases. The increase of salinity gradient reduces the heat losses from the hot source.

Future extensions of this work could be the application of the FS method to unstable configurations of THC. This will be useful to address the representativeness of 2D and 3D simulations with a high accurate solution free from discretization errors. The findings in this paper provide a technical support on the effect of pertinent parameters on three-dimensional THC, validity and applicability of these findings in real-word problem is worthy of future inquiry. This study has certain limitations. For example, existing extensive studies (Zhao et al., 2008b, 2016; Zhao, 2014) have demonstrated that chemical dissolution reactions can change both porosity and permeability of a porous medium and therefore affect the flow instability in the

porous medium. Chemical reactions were neglected in this study, even though they should be considered in the future studies.

\section{Acknowledgment}

Marwan Fahs would acknowledge the support from the National School of Water and Environmental Engineering of Strasbourg through the research project PORO6100. There is no data used in this modeling study. The code developed in this work is available on https://doi.org/10.5281/zenodo.4064644 


\section{References}

Alavyoon, F. (1993). On natural convection in vertical porous enclosures due to prescribed fluxes of heat and mass at the vertical boundaries. International Journal of Heat and Mass Transfer, 36(10), 2479-2498. https://doi.org/10.1016/S0017-9310(05)80188-7

Babaei, M., \& Islam, A. (2018). Convective- Reactive $\mathrm{CO}_{2}$ Dissolution in Aquifers With Mass Transfer With Immobile Water. Water Resources Research, 54(11), 9585-9604. https://doi.org/10.1029/2018WR023150

Bao, T., \& Liu, Z. (Leo). (2019). Thermohaline stratification modeling in mine water via doublediffusive convection for geothermal energy recovery from flooded mines. Applied Energy, 237, 566-580. https://doi.org/10.1016/j.apenergy.2019.01.049

Bennacer, R., Tobbal, A., Beji, H., \& Vasseur, P. (2001). Double diffusive convection in a vertical enclosure filled with anisotropic porous media. International Journal of Thermal Sciences, 40(1), 30-41. https://doi.org/10.1016/S1290-0729(00)01185-6

Bera, P., Pippal, S., \& Sharma, A. K. (2014). A thermal non-equilibrium approach on doublediffusive natural convection in a square porous-medium cavity. International Journal of Heat and Mass Transfer, 78, 1080-1094. https://doi.org/10.1016/j.ijheatmasstransfer.2014.07.041

Brown, P. N., Hindmarsh, A. C., \& Petzold, L. R. (1994). Using Krylov Methods in the Solution of Large-Scale Differential-Algebraic Systems. SIAM Journal on Scientific Computing, 15(6), 1467-1488. https://doi.org/10.1137/0915088

Cabeza, L. F., Martorell, I., Miró, L., Fernández, A. I., \& Barreneche, C. (2015). Introduction to thermal energy storage (TES) systems. In Advances in Thermal Energy Storage Systems (pp. 1-28). Elsevier. https://doi.org/10.1533/9781782420965.1

Chamkha, A. J. (2002). Double-diffusive convection in a porous enclosure with cooperating temperature and concentration gradients and heat generation or absorption effects. Numerical Heat Transfer, Part A: Applications, 41(1), 65-87. https://doi.org/10.1080/104077802317221447

Cooper, C. A., Glass, R. J., \& Tyler, S. W. (2001). Effect of buoyancy ratio on the development of double-diffusive finger convection in a Hele-Shaw Cell. Water Resources Research, 37(9), 2323-2332. https://doi.org/10.1029/2001WR000343

De Giorgio, G., Chieco, M., Zuffianò, L., Limoni, P., Sottani, A., Pedron, R., et al. (2018). The Compatibility of Geothermal Power Plants with Groundwater Dependent Ecosystems: The Case of the Cesine Wetland (Southern Italy). Sustainability, 10(2), 303. https://doi.org/10.3390/su10020303

Evans, D. G., Nunn, J. A., \& Hanor, J. S. (1991). Mechanisms driving groundwater flow near salt domes. Geophysical Research Letters, 18(5), 927-930. https://doi.org/10.1029/91GL00908

Fahs, M., Younes, A., \& Mara, T. A. (2014). A new benchmark semi-analytical solution for density-driven flow in porous media. Advances in Water Resources, 70, 24-35. https://doi.org/10.1016/j.advwatres.2014.04.013

Fahs, M., Younes, A., \& Makradi, A. (2015). A Reference Benchmark Solution for Free Convection in A Square Cavity Filled with A Heterogeneous Porous Medium. Numerical Heat Transfer, Part B: Fundamentals, 67(5), 437-462. https://doi.org/10.1080/10407790.2014.977183

Fahs, M., Ataie-Ashtiani, B., Younes, A., Simmons, C. T., \& Ackerer, P. (2016). The Henry problem: New semianalytical solution for velocity-dependent dispersion. Water 
Resources Research, 52(9), 7382-7407. https://doi.org/10.1002/2016WR019288

Guerrero-Martínez, F. J., Younger, P. L., Karimi, N., \& Kyriakis, S. (2017). Three-dimensional numerical simulations of free convection in a layered porous enclosure. International Journal of Heat and Mass Transfer, 106, 1005-1013. https://doi.org/10.1016/j.ijheatmasstransfer.2016.10.072

Hadidi, N., \& Bennacer, R. (2018a). Heat and mass transfer by natural convection in a bi-layered cubic enclosure with opposing temperature and concentration gradients. International Journal of Thermal Sciences, 132, 534-551. https://doi.org/10.1016/j.ijthermalsci.2018.06.013

Hadidi, N., \& Bennacer, R. (2018b). Heat and mass transfer by natural convection in a bi-layered cubic enclosure with opposing temperature and concentration gradients. International Journal of Thermal Sciences, 132, 534-551. https://doi.org/10.1016/j.ijthermalsci.2018.06.013

Henry, H. R. (1964). Effects of dispersion on salt encroachment in coastal aquifers, 1613-C, 7084.

Hughes, J. D., Sanford, W. E., \& Vacher, H. L. (2005). Numerical simulation of double-diffusive finger convection. Water Resources Research, 41(1). https://doi.org/10.1029/2003WR002777

Islam, A. W., Sharif, M. A. R., \& Carlson, E. S. (2013). Numerical investigation of double diffusive natural convection of $\mathrm{CO} 2$ in a brine saturated geothermal reservoir. Geothermics, 48, 101-111. https://doi.org/10.1016/j.geothermics.2013.07.001

Islam, A. W., Lashgari, H. R., \& Sephernoori, K. (2014). Double diffusive natural convection of $\mathrm{CO} 2$ in a brine saturated geothermal reservoir: Study of non-modal growth of perturbations and heterogeneity effects. Geothermics, 51, 325-336. https://doi.org/10.1016/j.geothermics.2014.03.001

Jafari Raad, S. M., Hassanzadeh, H., \& Ennis- King, J. (2019). On the Dynamics of Two- Component Convective Dissolution in Porous Media. Water Resources Research, 2018WR024572. https://doi.org/10.1029/2018WR024572

Jamshidzadeh, Z., Tsai, F. T.-C., Mirbagheri, S. A., \& Ghasemzadeh, H. (2013). Fluid dispersion effects on density-driven thermohaline flow and transport in porous media. Advances in Water Resources, 61, 12-28. https://doi.org/10.1016/j.advwatres.2013.08.006

Jamshidzadeh, Z., Tsai, F. T.-C., Ghasemzadeh, H., Mirbagheri, S. A., Barzi, M. T., \& Hanor, J. S. (2015). Dispersive thermohaline convection near salt domes: a case at Napoleonville Dome, southeast Louisiana, USA. Hydrogeology Journal, 23(5), 983-998. https://doi.org/10.1007/s10040-015-1251-4

Javaheri, M., Abedi, J., \& Hassanzadeh, H. (2010). Linear Stability Analysis of DoubleDiffusive Convection in Porous Media, with Application to Geological Storage of CO2. Transport in Porous Media, 84(2), 441-456. https://doi.org/10.1007/s11242-009-9513-z

Kalla, L., Vasseur, P., Benacer, R., Beji, H., \& Duval, R. (2001). Double diffusive convection within a horizontal porous layer salted from the bottom and heated horizontally. International Communications in Heat and Mass Transfer, 28(1), 1-10. https://doi.org/10.1016/S0735-1933(01)00207-X

Khadiri, A., Amahmid, A., Hasnaoui, M., \& Rtibi, A. (2010). Soret Effect on Double-Diffusive Convection in a Square Porous Cavity Heated and Salted from Below. Numerical Heat Transfer, Part A: Applications, 57(11), 848-868. https://doi.org/10.1080/10407782.2010.489487 
Koohbor, B., Fahs, M., Ataie-Ashtiani, B., Simmons, C. T., \& Younes, A. (2018). Semianalytical solutions for contaminant transport under variable velocity field in a coastal aquifer. Journal of Hydrology, 560, 434-450. https://doi.org/10.1016/j.jhydrol.2018.03.048

Langevin, C. D., Dausman, A. M., \& Sukop, M. C. (2010). Solute and Heat Transport Model of the Henry and Hilleke Laboratory Experiment. Ground Water, 48(5), 757-770. https://doi.org/10.1111/j.1745-6584.2009.00596.x

Le Lous, M., Larroque, F., Dupuy, A., \& Moignard, A. (2015). Thermal performance of a deep borehole heat exchanger: Insights from a synthetic coupled heat and flow model. Geothermics, 57, 157-172. https://doi.org/10.1016/j.geothermics.2015.06.014

Li, S., \& Petzold, L. (1999). Design of new DASPK for sensitivity analysis (Handbook of Systemic Autoimmune Diseases). Retrieved from https://www.researchgate.net/publication/2626137_Design_of_new_DASPK_for_sensiti vity_analysis/citations

van Lopik, J. H., Hartog, N., Zaadnoordijk, W. J., Cirkel, D. G., \& Raoof, A. (2015). Salinization in a stratified aquifer induced by heat transfer from well casings. Advances in Water Resources, 86, 32-45. https://doi.org/10.1016/j.advwatres.2015.09.025

Love, A. J., Simmons, C. T., \& Nield, D. A. (2007). Double-diffusive convection in groundwater wells. Water Resources Research, 43(8). https://doi.org/10.1029/2007WR006001

Magri, F., Akar, T., Gemici, U., \& Pekdeger, A. (2012). Numerical investigations of faultinduced seawater circulation in the Seferihisar-Balçova Geothermal system, western Turkey. Hydrogeology Journal, 20(1), 103-118. https://doi.org/10.1007/s10040-0110797-z

Mansour, A., Amahmid, A., Hasnaoui, M., \& Bourich, M. (2006). Multiplicity of Solutions Induced by Thermosolutal Convection in a Square Porous Cavity Heated from Below and Submitted to Horizontal Concentration Gradient in the Presence of Soret Effect. Numerical Heat Transfer, Part A: Applications, 49(1), 69-94. https://doi.org/10.1080/10407780500302166

Masuda, Y., Yoneya, M., \& Kimura, S. (2013). Multiple Solutions of Double-Diffusive Convection in Porous Media due to Opposing Heat and Mass Fluxes on Vertical Walls. Journal of Thermal Science and Technology, 8(3), 533-542. https://doi.org/10.1299/jtst.8.533

Mchirgui, A., Hidouri, N., Magherbi, M., \& Brahim, A. B. (2012). Entropy Generation in Double-Diffusive Convection in a Square Porous Cavity using Darcy-Brinkman Formulation. Transport in Porous Media, 93(1), 223-240. https://doi.org/10.1007/s11242-012-9954-7

Mohamad, A.A., \& Bennacer, R. (2002). Double diffusion, natural convection in an enclosure filled with saturated porous medium subjected to cross gradients; stably stratified fluid. International Journal of Heat and Mass Transfer, 45(18), 3725-3740. https://doi.org/10.1016/S0017-9310(02)00093-5

Mohamad, Abdulmajeed A., \& Bennacer, R. (2001). Natural convection in a confined saturated porous medium with horizontal temperature and vertical solutal gradients. International Journal of Thermal Sciences, 40(1), 82-93. https://doi.org/10.1016/S12900729(00)01182-0

Musuuza, J. L., Radu, F. A., \& Attinger, S. (2012). Predicting predominant thermal convection in thermohaline flows in saturated porous media. Advances in Water Resources, 49, 23- 
36. https://doi.org/10.1016/j.advwatres.2012.07.020

Navelot, V., Géraud, Y., Favier, A., Diraison, M., Corsini, M., Lardeaux, J.-M., et al. (2018). Petrophysical properties of volcanic rocks and impacts of hydrothermal alteration in the Guadeloupe Archipelago (West Indies). Journal of Volcanology and Geothermal Research, 360, 1-21. https://doi.org/10.1016/j.jvolgeores.2018.07.004

Nield, D. A., \& Kuznetsov, A. V. (2013). The Effect of Heterogeneity on the Onset of DoubleDiffusive Convection Induced by Internal Heating in a Porous Medium: A Layered Model. Transport in Porous Media, 100(1), 83-99. https://doi.org/10.1007/s11242-0130206-2

Nield, D. A., Simmons, C. T., Kuznetsov, A. V., \& Ward, J. D. (2008). On the evolution of salt lakes: Episodic convection beneath an evaporating salt lake. Water Resources Research, 44(2). https://doi.org/10.1029/2007WR006161

Peyret, R. (2013). Spectral Methods for Incompressible Viscous Flow. New York, NY: Springer.

Post, V., \& Abarca, E. (2010). Preface: Saltwater and freshwater interactions in coastal aquifers. Hydrogeology Journal, 18(1), 1-4. https://doi.org/10.1007/s10040-009-0561-9

van Reeuwijk, M., Mathias, S. A., Simmons, C. T., \& Ward, J. D. (2009). Insights from a pseudospectral approach to the Elder problem: PSEUDOSPECTRAL APPROACH TO THE ELDER PROBLEM. Water Resources Research, 45(4). https://doi.org/10.1029/2008WR007421

Schilling, O., Sheldon, H. A., Reid, L. B., \& Corbel, S. (2013). Hydrothermal models of the Perth metropolitan area, Western Australia: implications for geothermal energy. Hydrogeology Journal, 21(3), 605-621. https://doi.org/10.1007/s10040-012-0945-0

Segol, G. (1994). Classic groundwater simulations: proving and improving numerical models. Englewood Cliffs, N.J: PTR Prentice Hall.

Sezai, I., \& Mohamad, A. A. (1999). Three-dimensional double-diffusive convection in a porous cubic enclosure due to opposing gradients of temperature and concentration. Journal of Fluid Mechanics, 400, 333-353. https://doi.org/10.1017/S0022112099006540

Shao, Q., Fahs, M., Younes, A., \& Makradi, A. (2016). A high-accurate solution for DarcyBrinkman double-diffusive convection in saturated porous media. Numerical Heat Transfer, Part B: $\quad$ Fundamentals, $69(1), \quad$ 26-47. https://doi.org/10.1080/10407790.2015.1081044

Shao, Q., Fahs, M., Younes, A., Makradi, A., \& Mara, T. (2016). A new benchmark reference solution for double-diffusive convection in a heterogeneous porous medium. Numerical Heat Transfer, Part B: Fundamentals, 70(5), 373-392. https://doi.org/10.1080/10407790.2016.1215718

Shao, Q., Fahs, M., Hoteit, H., Carrera, J., Ackerer, P., \& Younes, A. (2018). A 3- D Semianalytical Solution for Density- Driven Flow in Porous Media. Water Resources Research, 54(12). https://doi.org/10.1029/2018WR023583

Sheldon, H. A., Florio, B., Trefry, M. G., Reid, L. B., Ricard, L. P., \& Ghori, K. A. R. (2012). The potential for convection and implications for geothermal energy in the Perth Basin, Western Australia. Hydrogeology Journal, 20(7), 1251-1268. https://doi.org/10.1007/s10040-012-0886-7

Simpson, Matthew J., \& Clement, T. P. (2004). Improving the worthiness of the Henry problem as a benchmark for density-dependent groundwater flow models: IMPROVING THE HENRY PROBLEM. Water Resources Research, 40(1). https://doi.org/10.1029/2003WR002199 
Simpson, M.J., \& Clement, T. P. (2003). Theoretical analysis of the worthiness of Henry and Elder problems as benchmarks of density-dependent groundwater flow models. Advances in Water Resources, 26(1), 17-31. https://doi.org/10.1016/S0309-1708(02)00085-4

Sivasankaran, S., Kandaswamy, P., \& Ng, C. O. (2008). Double diffusive convection of anomalous density fluids in a porous cavity. Transport in Porous Media, 71(2), 133-145. https://doi.org/10.1007/s11242-007-9114-7

Stajnko, J. K., Ravnik, J., \& Jecl, R. (2017). Numerical simulation of three-dimensional doublediffusive natural convection in porous media by boundary element method. Engineering Analysis with $\quad$ Boundary 69-79. https://doi.org/10.1016/j.enganabound.2016.12.007

Thorne, D., Langevin, C. D., \& Sukop, M. C. (2006). Addition of simultaneous heat and solute transport and variable fluid viscosity to SEAWAT. Computers \& Geosciences, 32(10), 1758-1768. https://doi.org/10.1016/j.cageo.2006.04.005

Trevisan, O. V., \& Bejan, A. (1986). Mass and heat transfer by natural convection in a vertical slot filled with porous medium. International Journal of Heat and Mass Transfer, 29(3), 403-415. https://doi.org/10.1016/0017-9310(86)90210-3

Vafai, K. (Ed.). (2015). Handbook of porous media (Third edition). Boca Raton: CRC Press, Taylor \& Francis Group.

Voss, C. I., Simmons, C. T., \& Robinson, N. I. (2010). Three-dimensional benchmark for variable-density flow and transport simulation: matching semi-analytic stability modes for steady unstable convection in an inclined porous box. Hydrogeology Journal, 18(1), 5-23. https://doi.org/10.1007/s10040-009-0556-6

Yilmaz Turali, E., \& Simsek, S. (2017). Conceptual and 3D simulation modeling of the Sorgun hydrothermal reservoir (Yozgat,Turkey). Geothermics, 66, 85-100. https://doi.org/10.1016/j.geothermics.2016.11.012

Younes, A., Fahs, M., \& Ackerer, P. (2007). A new approach to avoid excessive numerical diffusion in Eulerian-Lagrangian methods. Communications in Numerical Methods in Engineering, 24(11), 897-910. https://doi.org/10.1002/cnm.996

Younes, A., \& Fahs, M. (2014). A semi-analytical solution for saltwater intrusion with a very narrow transition zone. Hydrogeology Journal, 22(2), 501-506. https://doi.org/10.1007/s10040-014-1102-8

Younes, A., \& Fahs, M. (2015). Extension of the Henry semi-analytical solution for saltwater intrusion in stratified domains. Computational Geosciences, 19(6), 1207-1217. https://doi.org/10.1007/s10596-015-9534-3

Zhao, C., Hobbs, B. E., Ord, A., Peng, S., Mühlhaus, H. B., \& Liu, L. (2005). Double DiffusionDriven Convective Instability of Three-Dimensional Fluid-Saturated Geological Fault Zones Heated from Below. Mathematical Geology, 37(4), 373-391. https://doi.org/10.1007/s11004-005-5954-2

Zhao, C., Hobbs, B. E., \& Ord, A. (2008a). Convective and advective heat transfer in geological systems. Retrieved from https://doi.org/10.1007/978-3-540-79511-7

Zhao, C., Hobbs, B. E., Hornby, P., Ord, A., Peng, S., \& Liu, L. (2008b). Theoretical and numerical analyses of chemical-dissolution front instability in fluid-saturated porous rocks. International Journal for Numerical and Analytical Methods in Geomechanics, 32(9), 1107-1130. https://doi.org/10.1002/nag.661

Zhao, C., Hobbs, B. E., \& Ord, A. (2009). Fundamentals of computational geoscience: numerical methods and algorithms. Berlin; [New York]: Springer. 
Zhao, C., Hobbs, B. E., \& Ord, A. (2013). Theoretical analyses of acidization dissolution front instability in fluid-saturated carbonate rocks. International Journal for Numerical and Analytical Methods in Geomechanics, 37(13), 2084-2105. https://doi.org/10.1002/nag.2123

Zhao, C. (2014). Physical and Chemical Dissolution Front Instability in Porous Media. Cham: Springer International Publishing. https://doi.org/10.1007/978-3-319-08461-9

Zhao, C., Hobbs, B. E., \& Ord, A. (2015). Theoretical analyses of chemical dissolution-front instability in fluid-saturated porous media under non-isothermal conditions. International Journal for Numerical and Analytical Methods in Geomechanics, 39(8), 799-820. https://doi.org/10.1002/nag.2332

Zhao, C., Hobbs, B. E., \& Ord, A. (2016). Chemical dissolution-front instability associated with water-rock reactions in groundwater hydrology: Analyses of porosity-permeability relationship effects. Journal of Hydrology, 540, 1078-1087. https://doi.org/10.1016/j.jhydrol.2016.07.022

Zhao, C., Hobbs, B. E., \& Ord, A. (2018). Modeling of mountain topography effects on hydrothermal $\mathrm{Pb}-\mathrm{Zn}$ mineralization patterns: Generic model approach. Journal of Geochemical Exploration, 190, 400-410. https://doi.org/10.1016/j.gexplo.2018.04.004

Zechner, E., Dresmann, H., Mocuţa, M., Danchiv, A., Huggenberger, P., Scheidler, S., et al. (2019). Salt dissolution potential estimated from two-dimensional vertical thermohaline flow and transport modeling along a Transylvanian salt diapir, Romania. Hydrogeology Journal, 27(4), 1245-1256. https://doi.org/10.1007/s10040-018-1912-1

Zhu, Q. Y., Zhuang, Y. J., \& Yu, H. Z. (2017). Entropy generation due to three-dimensional double-diffusive convection of power-law fluids in heterogeneous porous media. International Journal of Heat and Mass Transfer, 106, 61-82. https://doi.org/10.1016/j.ijheatmasstransfer.2016.10.050

Zidane, A., Younes, A., Huggenberger, P., \& Zechner, E. (2012). The Henry semianalytical solution for saltwater intrusion with reduced dispersion. Water Resources Research, 48(6). https://doi.org/10.1029/2011WR011157 


\section{Appendix A: Coefficients of the spectral system}

$$
\begin{gathered}
\delta_{i}= \begin{cases}1 & \text { if } i=0 \\
0 & \text { if } i \neq 0\end{cases} \\
\alpha_{I}= \begin{cases}2 & \text { if } \mathrm{I}=0 \\
1 & \text { if } \mathrm{I} \neq 0\end{cases}
\end{gathered}
$$

$\Gamma_{G, r}= \begin{cases}\frac{1-(-1)^{G+r}}{G+r}+\frac{1-(-1)^{G-r}}{G-r} & \text { if } \mathrm{G} \neq r \\ 0 & \text { if } \mathrm{G}=r\end{cases}$

$A_{i, j, k}^{\prime}= \begin{cases}A_{i, j, k} & \text { if } i \leq N i, j \leq N j \text { and } k \leq N k \\ 0 & \text { else }\end{cases}$

$B_{i, j, k}^{\prime}= \begin{cases}B_{i, j, k} & \text { if } i \leq N l, j \leq N m \text { and } k \leq N n \\ 0 & \text { else }\end{cases}$

$E_{i, j, k}^{\prime}= \begin{cases}E_{i, j, k} & \text { if } i \leq N u, j \leq N v \text { and } k \leq N w \\ 0 & \text { else }\end{cases}$

$G_{i, j, k}^{\prime}= \begin{cases}G_{i, j, k} & \text { if } i \leq N s, j \leq N p \text { and } k \leq N t \\ 0 & \text { else }\end{cases}$

$\xi_{G, r, o}=\delta_{G, r+o}+\delta_{G, r-o}+\delta_{G,-r+o}+\delta_{G,-r-o}$

$\eta_{G, r, o}=\delta_{G, r+o}+\delta_{G, r-o}+\delta_{G,-r+o}$

$\gamma_{G, r, o}=\delta_{G, r+o}+\delta_{G, r-o}-\delta_{G,-r+o}$

$\kappa_{G, r, o}=\delta_{G, r+o}-\delta_{G, r-o}+\delta_{G,-r+o}$

$\zeta_{G, r, o}=-\delta_{G, r+o}+\delta_{G, r-o}+\delta_{G,-r+o}$

where $\delta_{i, j}$ is the Kronecker delta function 
Appendix B: converting the spectral equations to a system of ordinary differential equations

$$
\begin{aligned}
A_{I, J, K}= & \Upsilon_{I, J, K} \frac{N_{g} R a_{T} \alpha_{I}}{\pi} \sum_{v=1}^{N v} \sum_{w=0}^{N w} v E_{I, v, w}^{\prime} \Gamma_{J, v} \Gamma_{K, w} \\
& -\Upsilon_{I, J, K} \frac{2 N_{g} R a_{T}}{\pi} \Gamma_{J, 0} \Gamma_{K, 0} \delta_{I, 0}+\Upsilon_{I, J, K} \frac{R a_{T} J}{\pi} \sum_{s=1}^{N s} \sum_{t=0}^{N t} G_{s, J, t}^{\prime} \Gamma_{s, I} \Gamma_{K, t} \\
B_{L, M, N}= & \Upsilon_{M, L, N} \frac{L N_{g} R a_{T}}{\pi} \sum_{v=1}^{N v} \sum_{w=0}^{N w} E_{L, v, w}^{\prime} \Gamma_{v, M} \Gamma_{N, w} \\
& +\Upsilon_{M, L, N} \frac{R a_{T} \alpha_{M}}{\pi} \sum_{s=1}^{N s} \sum_{t=0}^{N t} s G_{s, M, t}^{\prime} \Gamma_{L, s} \Gamma_{N, t}-\Upsilon_{M, L, N} \frac{2 R a_{T}}{\pi^{2}} \Gamma_{L, 0} \Gamma_{N, 0} \delta_{M, 0}
\end{aligned}
$$

where $\Upsilon_{I, J, K}=\frac{-1}{\pi^{2}\left(I^{2}+\alpha_{I} J^{2}+\alpha_{I} K^{2}\right)}$ 


\section{Appendix C: Understanding the structure of the convective flow}

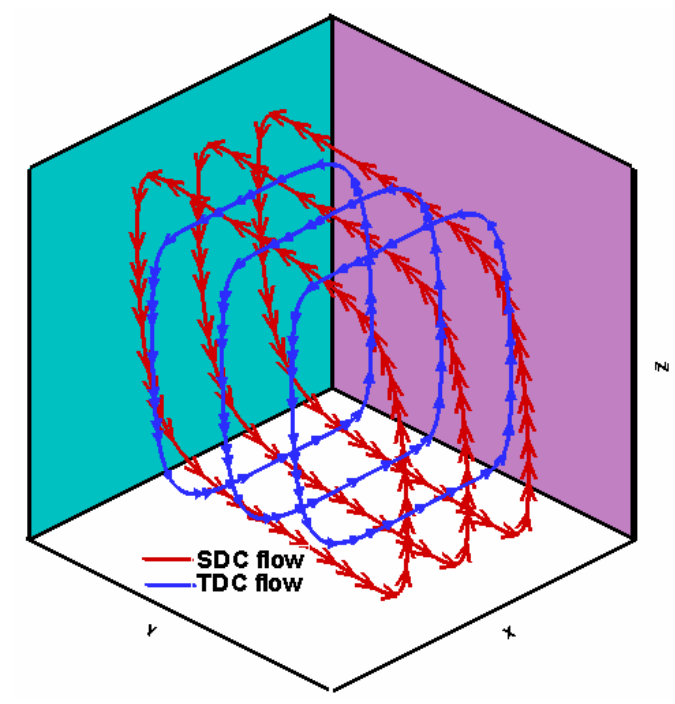

Figure C1. Components of the overall convective flow: The solute-driven convective flow (SDC) and the thermal-driven convective flow (TDC).
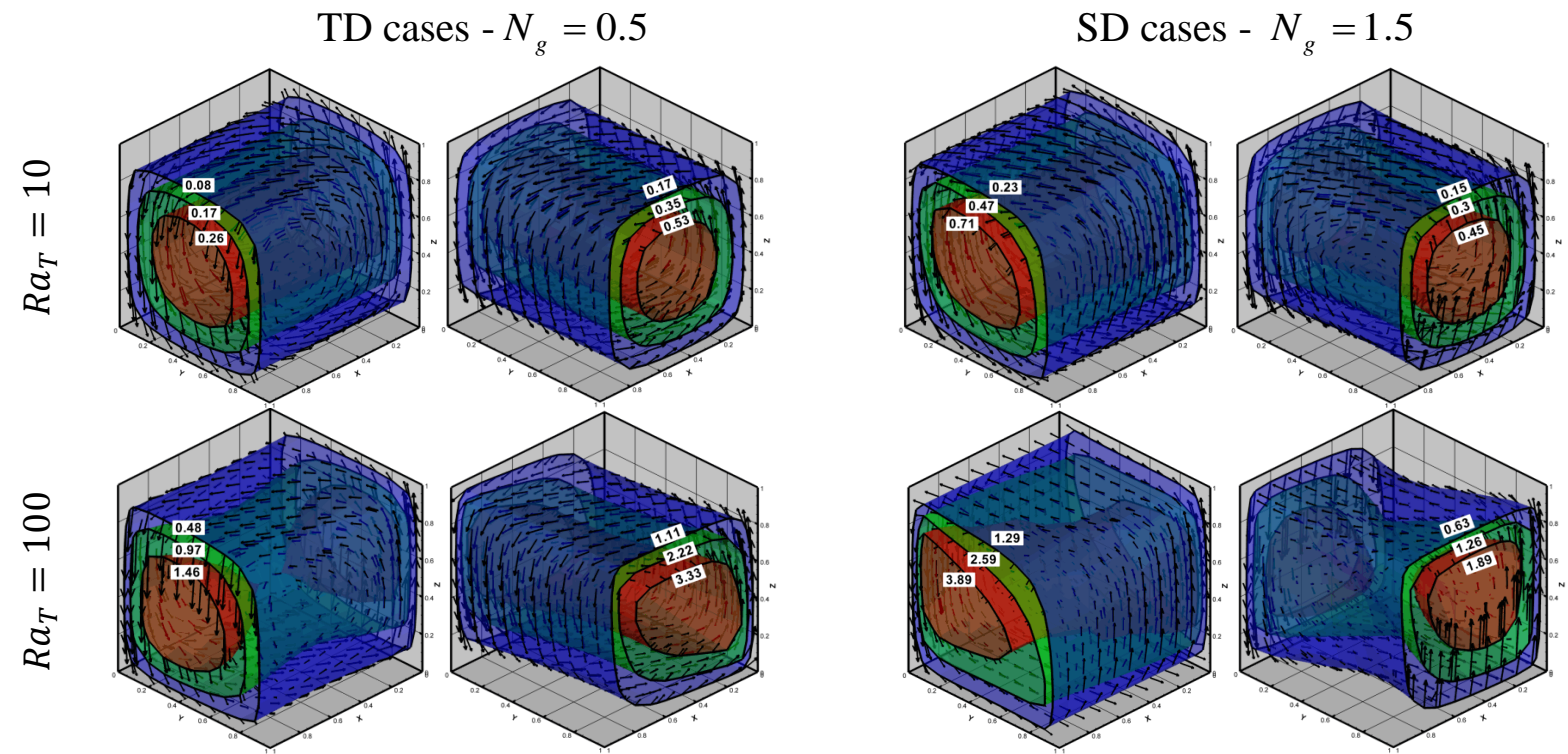

Figure C2. Steady-state isosurfaces of the vector potential components ( $\Psi_{X}$ : left and $\Psi_{Y}$ : right) and velocity field (arrows) at small and large thermal Rayleigh numbers in the cases of thermal (TD) and solute (SD) -dominated convective flow. 